\title{
Joint Physical Layer Coding and Network Coding for Bi-Directional Relaying
}

\author{
Makesh Pravin Wilson, Krishna Narayanan, Henry Pfister and Alex Sprintson
}

\begin{abstract}
We consider the problem of two transmitters wishing to exchange information through a relay in the middle. The channels between the transmitters and the relay are assumed to be synchronized, average power constrained additive white Gaussian noise channels with a real input with signal-to-noise ratio (SNR) of snr. An upper bound on the capacity is $\frac{1}{2} \log (1+\mathrm{snr})$ bits per transmitter per use of the medium-access phase and broadcast phase of the bi-directional relay channel. We show that using lattice codes and lattice decoding, we can obtain a rate of $\frac{1}{2} \log \left(\frac{1}{2}+\right.$ snr $)$ bits per transmitter, which is essentially optimal at high SNR. The main idea is to decode the sum of the codewords modulo a lattice at the relay followed by a broadcast phase which performs Slepian-Wolf coding with structured codes. For asymptotically low SNR, joint decoding of the two transmissions at the relay (MAC channel) is shown to be optimal. We also show that if the two transmitters use identical lattices with minimum angle decoding, we can achieve the same rate of $\frac{1}{2} \log \left(\frac{1}{2}+\mathrm{snr}\right)$. The proposed scheme can be thought of as a joint physical layer, network layer code which outperforms other recently proposed analog network coding schemes.
\end{abstract}

\section{Introduction, System Model And Problem STATEMEnt}

We consider the bi-directional relaying problem where two users try to exchange information with each other through a relay in the middle. More specifically, we study a simple 3-node linear Gaussian network as shown in Fig. 1. Nodes $A$ and $B$ wish to exchange information between each other through the relay node $R$, however, nodes $A$ and $B$ cannot communicate with each other directly. Let $\mathbf{u}_{A} \in\{0,1\}^{k}$ and $\mathbf{u}_{B} \in\{0,1\}^{k}$, be the information vectors at nodes $A$ and $B$ (vectors are denoted by bold face letters such as $\mathbf{u}$ throughout the paper). The information is assumed to be encoded into vectors (codewords) $\mathbf{x}_{1} \in \mathbb{R}^{n}$ and $\mathbf{x}_{2} \in \mathbb{R}^{n}$ at nodes $A$ and $B$, respectively, and transmitted. We assume that communication takes place in two phases - a multiple access (MAC) phase and a broadcast phase, which are briefly described below.

a) MAC phase: During the MAC phase, nodes $A$ and $B$ transmit $\mathbf{x}_{1}$ and $\mathbf{x}_{2}$ in $n$ uses of an AWGN channel to the relay. It is assumed that the two transmissions are perfectly synchronized and, hence, the received signal at the relay $\mathbf{y}_{R} \in \mathbb{R}^{n}$ is given by

$$
\mathbf{y}_{R}=\mathbf{x}_{1}+\mathbf{x}_{2}+\mathbf{z}
$$

where the components of $\mathbf{z}$ are independent identically distributed (i.i.d) Gaussian random variables with zero mean and variance $\sigma^{2}$. Further, it is assumed that there is an average transmit power constraint of $P$ at both nodes and, hence, $E\left[\left\|\mathbf{x}_{1}\right\|^{2}\right] \leq n P$ and $E\left[\left\|\mathbf{x}_{2}\right\|^{2}\right] \leq n P$.

b) Broadcast phase: During the broadcast phase, the relay node transmits $\mathbf{x}_{R} \in \mathbb{R}^{n}$ in $n$ uses of an AWGN broadcast channel to both nodes $A$ and $B$. It is assumed that the average transmit power at the relay node is also constrained to $P$ and that the noise variance at the two nodes is also $\sigma^{2}$. Node $A$ forms an estimate of $\mathbf{u}_{B}$, namely $\hat{\mathbf{u}}_{B}$ and node $B$ forms an estimate of $\mathbf{u}_{A}$, namely $\hat{\mathbf{u}}_{A}$. An error is said to occur if either $\mathbf{u}_{B} \neq \hat{\mathbf{u}}_{B}$ or $\mathbf{u}_{A} \neq \hat{\mathbf{u}}_{A}$, i.e., the probability of error is given by

$$
P_{e} \triangleq \operatorname{Pr}\left(\left\{\mathbf{u}_{A} \neq \hat{\mathbf{u}}_{A}\right\} \bigcup\left\{\mathbf{u}_{B} \neq \hat{\mathbf{u}}_{B}\right\}\right)
$$

It is assumed that the communications in the MAC and broadcast phases are orthogonal. For example, the communications in the MAC and broadcast phase could be in two separate frequency bands (or in two different time slots) and, hence, the MAC phase and broadcast phase do not interfere with each other. To keep the discussion simple, we will assume that the MAC and broadcast phases occur in different time slots. This can be easily

This work was supported by the National Science Foundation under grant CCR-0515296. The authors are with the Department of Electrical and Computer Engineering, Texas A\&M University, College Station, TX 77843, USA $\{$ makesh, krn, hpfister, spalex\}@ece.tamu.edu 

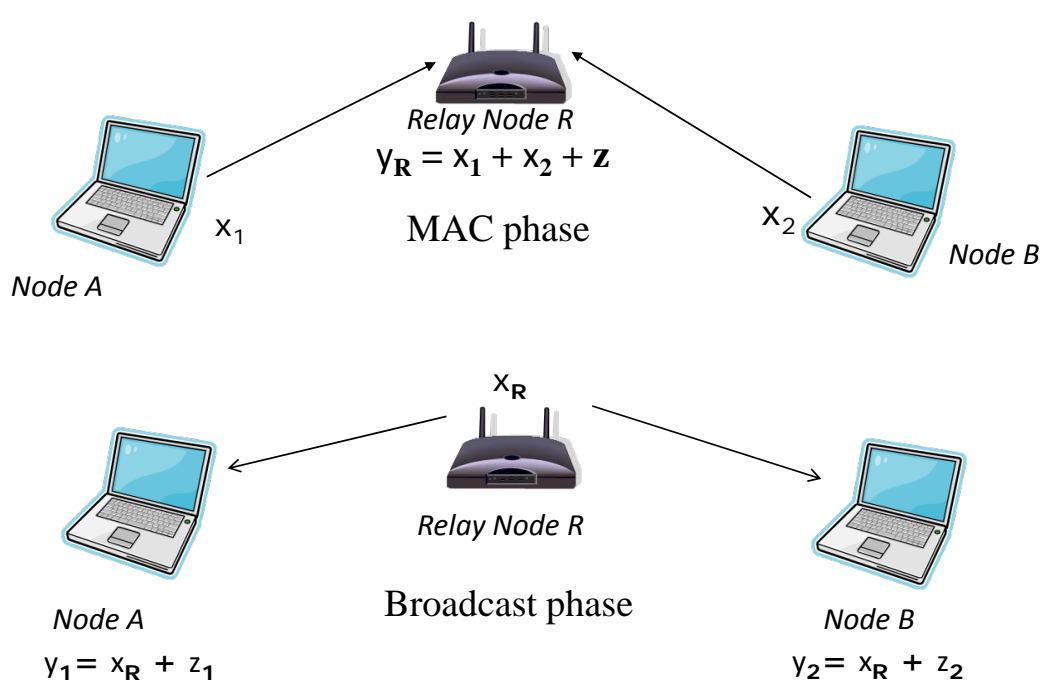

Fig. 1. System Model with 3 Nodes

generalized to the case when $2 n$ dimensions are available for communication, out of which $n$ dimensions are allocated to the MAC phase and $n$ dimensions are allocated to the broadcast phase. Note that the signal-to-noise ratio (SNR) for all transmissions is snr $=P / \sigma^{2}$ and, hence, we refer to it as simply the SNR without having to distinguish between the SNRs of the different phases. Similarly, we restrict our attention to the case when the both nodes $A$ and $B$ wish to exchange identical amount of information and, hence, we can simply refer to one exchange rate without having to distinguish between the rate for $A$ and $B$ separately.

Formally, we define the exchange rate $R_{e x, s c h e m e}$ for an encoding/decoding scheme as the maximum information rate that can be exchanged reliably, i.e.,

$$
R_{\text {ex }, \text { scheme }} \triangleq \max \frac{k}{n}: P_{e} \rightarrow 0 \quad \text { as } \quad n \rightarrow \infty .
$$

The exchange capacity $C_{e x}$ is then the supremum of $R_{e x, \text { scheme }}$ over all possible encoding/decoding schemes.

\section{MAIN RESUltS AND COMMENTS}

We mainly consider the case when the MAC and broadcast phase are both restricted to using exactly $n$ uses of the channel each. For this case, the main results in this paper are

- The exchange capacity satisfies

$$
C_{e x} \leq \frac{1}{2} \log \left(1+\frac{P}{\sigma^{2}}\right)
$$

because the MAC and broadcast phase each consist of $n$ AWGN channel uses.

- An exchange rate of

$$
R_{e x, \text { Lattice }}=\frac{1}{2} \log \left(\frac{1}{2}+\frac{P}{\sigma^{2}}\right)
$$

is achievable using the lattice coding scheme with dither and lattice decoding discussed in Section VI. The same exchange rate can also be obtained using the lattice coding scheme (without dither) and minimum angle decoding discussed in Section VII. At high SNR, these lattice based coding and decoding schemes are nearly optimal because their rates approach the upper bound. 
- An exchange rate of

$$
R_{e x, J D}=\frac{1}{4} \log \left(1+\frac{2 P}{\sigma^{2}}\right)
$$

is achievable using the joint decoding scheme in Section VIII. At low SNR, these scheme is nearly optimal because the rate approaches the upper bound.

- Clearly, any rate of the form $\beta R_{e x, J D}+(1-\beta) R_{e x, \text { Lattice }}$ is achievable for any $0 \leq \beta \leq 1$ by time sharing between the two schemes. This outperforms the recently proposed analog network coding idea in [3] over the entire range of SNR.

\section{RELATED PRIOR WORK}

Recently, there has been a significant amount of work on coding for the bi-directional relay problem [1]-[7]. In [2], Katti et al., showed the usefulness of network coding for this problem. Although they do not consider the physical layer explicitly in this work, the natural extension of their solution to our problem would work as follows. The $2 n$ channel uses available for signaling would be split into three slots with $2 n / 3$ channel uses each. In the first time slot, $\mathbf{u}_{A}$ is encoded using an optimal channel code for the AWGN channel into $\mathbf{x}_{1}$ and transmitted from node $A$. Similarly, in the second time slot $\mathbf{u}_{B}$ is encoded into $\mathbf{x}_{2}$ and transmitted from node $B$. At the relay, $\mathbf{u}_{A}$ and $\mathbf{u}_{B}$ are decoded and then the relay forms $\mathbf{u}_{R}=\mathbf{u}_{A} \oplus \mathbf{u}_{B}$ and encodes $\mathbf{u}_{R}$ into $\mathbf{x}_{R}$ using an optimal code for the Gaussian channel and broadcasts into both nodes. The two nodes decode $\mathbf{u}_{R}$ and then since they have $\mathbf{u}_{A}$ and $\mathbf{u}_{B}$, they can obtain $\mathbf{u}_{B}$ and $\mathbf{u}_{A}$ at the nodes $A$ and $B$, respectively. Here, the physical layer and network layer are completely separated and coding (or mixing of the information) is performed only at the network layer. In the system model considered in Fig. 1, the physical layer naturally performs mixing of the signals from the two transmitters. The schemes that take advantage of this can be referred to as joint physical layer coding and network coding solutions.

One such scheme called analog network coding was recently proposed in [3]. In this case, the MAC phase and broadcast phase use $n$ uses of the AWGN and are orthogonal to each other. Gaussian code books are used at the transmitters to encode $\mathbf{u}_{A}$ into $\mathbf{x}_{1}$ and $\mathbf{u}_{B}$ into $\mathbf{x}_{2}$, respectively. Analog network coding is an amplify (rather than scale) and forward scheme where the received signal at the relay during the MAC phase $\mathbf{y}_{R}$, is scaled to satisfy the power constraint and transmitted during the broadcast phase, i.e., $\mathbf{x}_{R}=\sqrt{\frac{P}{2 P+\sigma^{2}}} \mathbf{y}_{R}$. It can be seen that this scheme can achieve an exchange capacity of $\frac{1}{2} \log \left(1+\frac{P / \sigma^{2}}{\frac{3 P+\sigma^{2}}{P}}\right)$, which is higher than that achievable with the pure network coding scheme in [2] for high SNR.

The schemes proposed in this paper can be thought of as decode and forward schemes which outperform the amplify and forward scheme in [3]. In a very recent work [6], it is conjectured that an exchange rate of $\frac{1}{2} \log \left(1+\frac{P}{\sigma^{2}}\right)$ can be achieved, however, no scheme is given or even conjectured. The scheme in this paper is a constructive scheme that performs close to the rate conjectured in [6]. The lattice decoding scheme discussed in Section VI is similar to that used by Nazer and Gastpar [20] for the problem of estimation the sum of two Gaussian random variables, but was independently proposed in the previous version of this paper [19].

\section{An Optimal Transmission Scheme for the BSC Channel}

To motivate our proposed scheme, we first consider a system where the physical layer channels are all binary symmetric channels. i.e., $\mathbf{x}_{1} \in\{0,1\}^{n}, \mathbf{x}_{2} \in\{0,1\}^{n}$ and the signal received at the relay is

$$
\mathbf{y}_{R}=\mathbf{x}_{1} \oplus \mathbf{x}_{2} \oplus \mathbf{e}
$$

where $\oplus$ denotes binary addition and $\mathbf{e}$ is an error sequence whose components are 0 or 1 with probability $q$ and $1-q$ respectively and are i.i.d. Similarly, during the broadcast phase also let the channel be a BSC channel with crossover probability $q$. In this case, an upper bound on the exchange capacity can be seen to be $1-H(q)$ since this is the maximum information that can flow to any of the nodes from the relay. This can be achieved using the following coding scheme.

In this scheme, the two nodes use identical capacity achieving binary linear codes of rate $1-H(q)$. Consider again the received signal at the relay given in (1). Notice that since $\mathbf{x}_{1}$ and $\mathbf{x}_{2}$ are codewords of the same linear code, $\mathbf{x}_{1} \oplus \mathbf{x}_{2}$ is also a valid codeword from the same code which achieves capacity over a BSC channel with 
crossover probability $q$. Hence, the relay can decode $\mathbf{x}_{1} \oplus \mathbf{x}_{2}$ and transmit the result during the broadcast phase. The nodes $A$ and $B$ can also decode $\mathbf{x}_{1} \oplus \mathbf{x}_{2}$ and since they have $\mathbf{x}_{1}$ and $\mathbf{x}_{2}$, they can obtain $\mathbf{x}_{2}$ and $\mathbf{x}_{1}$, respectively. This scheme achieves an exchange rate of $1-H(q)$ and is therefore optimal.

Random codes versus structured codes: It is quite interesting to note that if random codes, i.e., codes from the Shannon ensemble were used instead of linear codes, $\mathbf{x}_{1} \oplus \mathbf{x}_{2}$ cannot be decoded at the relay. The linearity (or group structure) of the code is exploited to make $\mathbf{x}_{1} \oplus \mathbf{x}_{2}$ decodable at the relay and, hence, structured codes with a group structure outperform random codes for this problem. Examples of schemes were structured codes outperform random codes have been given in Korner and Marton [8] and more recently by Nazer and Gastpar in [9], [10].

\section{UPPER BOUND ON THE EXCHANGE RATE FOR GAUSSIAN LINKS}

Let us now return to the problem outlined in Section I] where the channels between the nodes and the relay are AWGN channels. We restrict our attention to schemes where the MAC phase and broadcast phase are both orthogonal to each other and use $n$ channel uses (or dimensions). With this restriction, a simple upper bound on the exchange rate can be obtained as follows. Consider a cut between the relay node and node $A$. The maximum amount of information that can flow to either of the nodes from the relay is $\frac{1}{2} \log \left(1+\frac{P}{\sigma^{2}}\right)$. Hence, the exchange capacity is upper bounded by

$$
C_{e x, u b}=\frac{1}{2} \log \left(1+\frac{P}{\sigma^{2}}\right) .
$$

We now consider coding schemes and analyze their performance.

\section{Nested Lattice Based Coding Scheme with Lattice Decoding}

As shown in Section IV for the BSC channel, codes with a group structure (linear codes) enable decoding of a linear combination (or, sum) codewords at the relay. This motivates the use of lattice codes for the Gaussian channel since lattices have a similar group structure with respect to real vector addition. We begin with some preliminaries about lattices [12], [14].

An $n$-dimensional lattice $\Lambda$ is a subgroup of $\mathbb{R}^{n}$ under vector addition over the reals. This implies that if $\lambda_{1}, \lambda_{2} \in \Lambda$, then $\lambda_{1}+\lambda_{2} \in \Lambda$. For any $\mathbf{x} \in \mathbb{R}^{n}$, the quantization of $\mathbf{x}, Q_{\Lambda}(\mathbf{x})$ is defined as the $\lambda \in \Lambda$ that is closest to $\mathbf{x}$ with respect to Euclidean distance. The fundamental Voronoi region $\mathcal{V}(\Lambda)$ is defined as $\mathcal{V}(\Lambda)=\left\{\mathbf{x}: Q_{\Lambda}(\mathbf{x})=\mathbf{0}\right\}$. The mod operation is defined as $(\mathbf{x} \bmod \Lambda)=\mathbf{x}-Q_{\Lambda}(\mathbf{x})$. This can be interpreted as the error in quantizing an $\mathrm{x}$ to the closest point in the lattice $\Lambda$. The second moment of a lattice is given by

$$
\sigma^{2}(\Lambda)=\frac{1}{V(\Lambda)} \frac{1}{n} \int_{\mathcal{V}(\Lambda)}\|\mathbf{x}\|^{2} d \mathbf{x}
$$

and where $V(\Lambda)$, the volume of the fundamental Voronoi region is denoted by $V(\Lambda)=\int_{\mathcal{V}(\Lambda)} d \mathbf{x}$. The normalized second moment of the lattice is then given by

$$
G(\Lambda)=\sigma^{2}(\Lambda) / V(\Lambda)^{1 / n}
$$

Let us define the covering radius of a lattice $\mathcal{R}_{u}$ as the radius of the smallest $n$-dimensional hyper sphere containing the Voronoi region $\mathcal{V}(\Lambda)$. Also let $\mathcal{R}_{l}$ denote the effective radius of $\mathcal{V}(\Lambda)$, which is the radius of the $n$-dimensional hyper sphere having the same volume as $\mathcal{V}(\Lambda)$. Now we can define a Rogers-good Lattice [12, (69)] as

$$
1 \leq\left(\frac{\mathcal{R}_{u}}{\mathcal{R}_{l}}\right)^{n}<c \cdot n \cdot(\log n)^{a},
$$

where $c$ and $a$ are constants. This implies,

$$
\frac{1}{n} \log \frac{\mathcal{R}_{u}}{\mathcal{R}_{l}}=\mathcal{O}\left(\frac{1}{n}\right)
$$

In this work, we are interested in nested lattices. Formally, we can say the lattice $\Lambda_{c}$ (the coarse lattice) is nested in the lattice $\Lambda_{f}$ (the fine lattice) if $\Lambda_{c} \subseteq \Lambda_{f}$ [13]. Let the fundamental Voronoi regions of the lattices, $\Lambda_{c}$ and $\Lambda_{f}$ be $\mathcal{V}\left(\Lambda_{c}\right)$ and $\mathcal{V}\left(\Lambda_{f}\right)$. The existence of nested lattices for which $G\left(\Lambda_{c}\right) \approx 1 /(2 \pi e)$ and $G\left(\Lambda_{f}\right) \approx 1 /(2 \pi e)$ has 


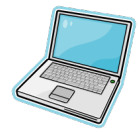

Node A

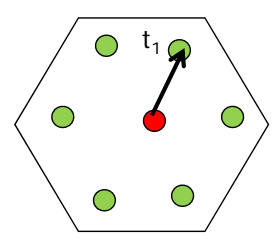

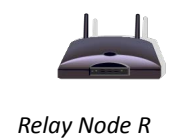

Relay Node $R$

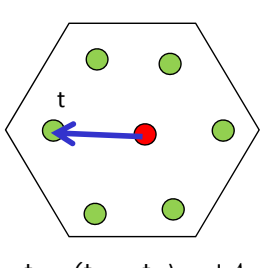

$\mathrm{t}=\left(\mathrm{t}_{1}+\mathrm{t}_{2}\right) \bmod \Lambda$
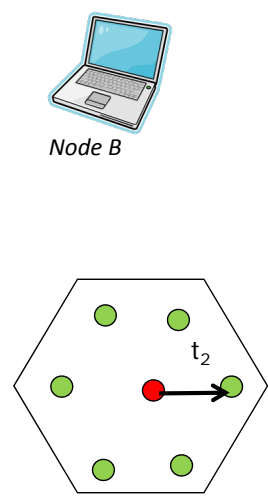

Fig. 2. Lattice code based scheme showing the transmitted signals $\mathbf{t}_{1}, \mathbf{t}_{2}$ and the decoded signal at the relay $\mathbf{t}$

been shown in [13], [14]. The number of lattice points of the fine lattice in the basic Voronoi region of the coarse lattice is given by the nesting ratio $\frac{V\left(\Lambda_{c}\right)}{V\left(\Lambda_{f}\right)}$.

Lattice codes can be used to achieve capacity on the single user AWGN channel under maximum likelihood (ML) [15], [17], [11]. More recently, nested lattices have been shown to achieve the capacity of the single user AWGN channel under lattice decoding [12], [14]. The main idea in [12], [14] is to use the coarse lattice as a shaping region and the lattice points from the fine lattice contained within the basic Voronoi region of the coarse lattice as the codewords. The existence of good nested lattices that achieve capacity has been shown in [12].

\section{A. Description}

We now describe our encoding and decoding schemes for the bi-directional relaying problem using nested lattices. The encoding and decoding operations during the MAC and broadcast phase are explained below. A general schematic is also shown in Fig. 2 .

MAC Phase: Let there be $k$ information bits in the information vector $\mathbf{u}_{A}$ and $\mathbf{u}_{B}$ and, hence, the exchange rate is $R=k / n$. At node $A$, the information vector $\mathbf{u}_{A}$ is mapped onto a fine lattice point $\mathbf{t}_{1} \in\left\{\Lambda_{f} \cap \mathcal{V}\left(\Lambda_{c}\right)\right\}$, i.e., the set of all fine lattice points in the basic Voronoi region of the coarse lattice is taken to be the code. An identical code is used at node $B$ and the information vector $\mathbf{u}_{B}$ is mapped onto the codeword $\mathbf{t}_{2} \in\left\{\Lambda_{f} \cap \mathcal{V}\left(\Lambda_{c}\right)\right\}$. We then generate dither vectors $\mathbf{d}_{1}$ and $\mathbf{d}_{2}$ which are randomly generated $n$ dimensional vectors uniformly distributed over $\mathcal{V}\left(\Lambda_{c}\right)$. The dither vectors are mutually independent of each other and are known at both the relay node and the nodes $A$ and $B$. Now node $A$ and node $B$ form the transmitted signal $\mathbf{x}_{1}$ and $\mathrm{x}_{2}$ as follows

$$
\begin{array}{ll}
\mathbf{x}_{1}=\left(\mathbf{t}_{1}-\mathbf{d}_{1}\right) & \bmod \Lambda_{c} \\
\mathbf{x}_{2}=\left(\mathbf{t}_{2}-\mathbf{d}_{2}\right) & \bmod \Lambda_{c}
\end{array}
$$

By choosing an appropriate coarse lattice with second moment $P$, the transmit power constraint will be satisfied at both nodes. Assuming perfect synchronization, the relay node receives $\mathbf{y}_{R}$ given by

$$
\mathbf{y}_{R}=\mathbf{x}_{1}+\mathbf{x}_{2}+\mathbf{z}
$$

where $\mathbf{z}$ is the noise vector whose components have variance $\sigma^{2}$.

The main idea is that the relay decodes

$$
\mathbf{t}=\left(\mathbf{t}_{1}+\mathbf{t}_{2}\right) \bmod \Lambda_{c}
$$

from the received signal $\mathbf{y}_{R}$. 
Broadcast Phase: In the broadcast phase, the relay node transmits the index of $\mathbf{t}$ using a capacity achieving code for the AWGN channel. The index (or, equivalently, t) can be obtained at the nodes $A$ and $B$, and using a $\bmod \Lambda$ operation, $\mathbf{t}_{2}$ and $\mathbf{t}_{1}$ can can be recovered at nodes $A$ and $B$, respectively. This scheme can also be thought of as a decode and forward scheme where the relay decodes a function of $\mathbf{t}_{1}$ and $\mathbf{t}_{2}$, namely $\mathbf{t}=\left(\mathbf{t}_{1}+\mathbf{t}_{2}\right) \bmod \Lambda_{c}$ and forwards this to the nodes. Notice however, that the relay will not know either $\mathbf{t}_{1}$ or $\mathbf{t}_{2}$ exactly, it only knows $\mathbf{t}$.

\section{B. Achievable rate}

Before we discuss the achievable exchange rate with the lattice encoding scheme and decoding scheme discussed above, we need a few definitions and lemmas.

The coding rate of the nested lattice code is defined as the logarithm of the number of lattice points of the fine lattice in $\Lambda_{f} \cap \mathcal{V}\left(\Lambda_{c}\right)$ which is given by

$$
\frac{1}{n} \log \left|\Lambda_{f} \cap \mathcal{V}\left(\Lambda_{c}\right)\right|=\frac{1}{n} \log \frac{V\left(\Lambda_{c}\right)}{V\left(\Lambda_{f}\right)}
$$

Lemma 1: Let $t_{1}$ and $t_{2}$ be independent random variables which are uniformly distributed over the set of all fine lattice points in $\mathcal{V}\left(\Lambda_{c}\right)$, i.e., $\operatorname{Pr}\left[t_{1}=\lambda\right]=1 / 2^{n R}, \forall \lambda \in\left\{\Lambda_{f} \cap \mathcal{V}\left(\Lambda_{c}\right)\right\}$ and $\operatorname{Pr}\left[t_{2}=\lambda\right]=1 / 2^{n R}, \forall \lambda \in\left\{\Lambda_{f} \cap \mathcal{V}\left(\Lambda_{c}\right)\right\}$, where $R$ is the coding rate of the nested lattice code. Then, $t=\left(t_{1}+t_{2}\right) \bmod \Lambda$ is also uniformly distributed over $\left\{\Lambda_{f} \cap \mathcal{V}\left(\Lambda_{c}\right)\right\}$.

\section{Proof:}

$$
\begin{aligned}
\operatorname{Pr}[t=\lambda] & =\sum_{i=1}^{2^{n R}} \operatorname{Pr}\left[t_{1}=\lambda_{i}\right] \operatorname{Pr}\left[t=\lambda \mid t_{1}=\lambda_{i}\right] \\
& =\sum_{i=1}^{2^{n R}} \frac{1}{2^{n R}} \operatorname{Pr}\left[\left(\lambda_{i}+t_{2}\right) \quad \bmod \Lambda_{c}=\lambda \mid t_{1}=\lambda_{i}\right] \\
& =\sum_{i=1}^{2^{n R}} \frac{1}{2^{n R}} \operatorname{Pr}\left[t_{2}=\left(\lambda-\lambda_{i}\right) \quad \bmod \Lambda_{c} \mid t_{1}=\lambda_{i}\right] \\
& =\sum_{i=1}^{2^{n R}} \frac{1}{2^{n R}} \frac{1}{2^{n R}}=\frac{1}{2^{n R}}
\end{aligned}
$$

We restate some of definitions in [12]. Let $\mathcal{R}_{u}$ be the covering radius of $\Lambda_{c}$ and let $\mathcal{B}\left(\mathcal{R}_{u}\right)$ be the $n$-dimensional ball of radius $\mathcal{R}_{u}$. Let $\rho^{2}$ be the second moment (per dimension) of the smallest ball containing $\mathcal{V}\left(\Lambda_{c}\right)$, i.e.,

$$
\rho^{2}=\frac{1}{n} \frac{1}{\left\|\mathcal{B}\left(\mathcal{R}_{u}\right)\right\|} \int_{\mathcal{B}\left(\mathcal{R}_{u}\right)}\|\mathbf{u}\|^{2} d \mathbf{u} .
$$

Note that $\mathcal{V}\left(\Lambda_{c}\right)$ has a second moment $P$ and hence $P<\rho^{2}$. Let $\mathbf{Z}^{*}$ be a random variable given by $\mathbf{Z}^{*}=$ $(1-\alpha)\left(\mathbf{Z}_{\mathbf{1}}+\mathbf{Z}_{\mathbf{2}}\right)+\alpha \mathbf{Z}$, with $\mathbf{Z}_{\mathbf{1}} \sim \mathcal{N}\left(0, \rho^{2} \mathbf{I}^{n}\right), \mathbf{Z}_{\mathbf{2}} \sim \mathcal{N}\left(0, \rho^{2} \mathbf{I}^{n}\right)$ and $\mathbf{Z} \sim \mathcal{N}\left(0, \sigma^{2} \mathbf{I}^{n}\right)$, where $\mathbf{I}^{n}$ is the $n \times n$ identity matrix. The variance of $\mathbf{Z}^{*}$ satisfies

Lemma 2 (Modified version of [12, Lemma 6]):

$$
\frac{n}{n+2} \cdot \frac{2 P \sigma^{2}}{2 P+\sigma^{2}} \leq \operatorname{Var}\left(\mathrm{Z}^{*}\right)=(1-\alpha)^{2}\left(\rho^{2}+\rho^{2}\right)+\alpha^{2} \sigma^{2} \leq\left(\frac{R_{u}}{R_{l}}\right)^{2} \frac{2 P \sigma^{2}}{2 P+\sigma^{2}},
$$

where $\mathcal{R}_{u}$ is the covering radius of the coarse lattice $\Lambda_{c}$ and $\mathcal{R}_{l}$ is the radius of the $n$-dimensional hyper sphere whose volume is equal to the volume of the basic Voronoi region $\mathcal{V}\left(\Lambda_{c}\right)$. 
Proof: The proof of the above lemma closely follows the proof in [12]. Equations [12, 116-118] can be used to get a bound on $\operatorname{Var}\left(Z_{1}\right)$ and $\operatorname{Var}\left(Z_{2}\right)$, i.e., $\rho^{2}$. Choosing $\alpha=\frac{2 P}{2 P+\sigma^{2}}$, the modified version of [12, Lemma 6] can be proved.

Let $\mathbf{X}_{1}$ and $\mathbf{X}_{2}$ be two independent random variables which are uniformly distributed over $\mathcal{V}\left(\Lambda_{c}\right)$ and let $\mathbf{Z} \sim \mathcal{N}\left(\mathbf{0}, \sigma^{2} \mathbf{I}\right)$ be an $n$-dimensional Gaussian vector independent of $\mathbf{X}_{1}$ and $\mathbf{X}_{2}$. Further, let $\mathbf{Z}_{e q}=(1-\alpha)\left(\mathbf{X}_{1}+\right.$ $\left.\mathbf{X}_{2}\right)+\alpha \mathbf{Z}$, where $\alpha=\frac{2 P}{2 P+\sigma^{2}}$. Notice that $\mathbf{Z}_{e q}$ is not Gaussian. We next state a Lemma which is a modified version of [12, Lemma 11] by Erez and Zamir which essentially shows that there exist good lattices for which $\mathbf{Z}_{e q}$ can be well approximated by a Gaussian of nearly the same variance and the approximation gets better as $n \rightarrow \infty$.

Lemma 3 (Modified version of [12, Lemma 11]): Let $\Lambda_{c}$ be a lattice which is both Rogers-good as well as Poltyrev-good. Then, for any $\mathbf{x}$,

$$
f_{Z_{e q}}(\mathbf{x})<e^{\epsilon_{1}\left(\Lambda_{c}\right) n} f_{\mathbf{Z}^{*}}(\mathbf{x}),
$$

where $\epsilon_{1}\left(\Lambda_{c}\right) \rightarrow 0$ as $n \rightarrow \infty$.

Proof: To prove the modified version of [12, Lemma 11], we can repeat the steps in [12], and equation [12, $200]$ can be restated with the notation in this paper as

Similarly for $\mathbf{Z}_{2}$, we get

$$
\frac{1}{n} \log \frac{f_{\mathbf{x}_{1}}(\mathbf{x})}{f_{\mathbf{Z}_{1}}(\mathbf{x})}=\epsilon_{1}\left(\Lambda_{c}\right)=\mathcal{O}\left(\frac{1}{n}\right) .
$$

$$
\frac{1}{n} \log \frac{f_{\mathbf{X}_{\mathbf{2}}}(\mathbf{x})}{f_{\mathbf{Z}_{\mathbf{2}}}(\mathbf{x})}=\epsilon_{1}\left(\Lambda_{c}\right)=\mathcal{O}\left(\frac{1}{n}\right) .
$$

Combining (14) and $(15)$ and also the definition of $\mathbf{Z}^{*}$ as $\mathbf{Z}^{*}=(1-\alpha)\left(\mathbf{Z}_{\mathbf{1}}+\mathbf{Z}_{\mathbf{2}}\right)+\alpha \mathbf{N}$, we can get the proof of the modified version of [12, Lemma 11].

We next state the theorem which is an application of the above Lemmas. This is very similar to [12, Theorem 5].

Theorem 4 (modified version of [12, Theorem 5]): Let $\mathbf{x}_{1}$ and $\mathbf{x}_{2}$ be realizations of two independent random variables which are uniformly distributed over $\mathcal{V}\left(\Lambda_{c}\right)$ and let $\mathbf{z}$ be a realization of an $n$-dimensional Gaussian vector $\mathbf{Z} \sim \mathcal{N}\left(\mathbf{0}, \sigma^{2} \mathbf{I}\right)$. Further, let $\mathbf{z}_{e q}=(1-\alpha)\left(\mathbf{x}_{1}+\mathbf{x}_{2}\right)+\alpha \mathbf{z}$, where $\alpha=\frac{2 P}{2 P+\sigma^{2}}$. For any coding rate $R<\frac{1}{2} \log \left(\frac{1}{2}+\frac{P}{\sigma^{2}}\right)$, there exists a sequence of $n$-dimensional nested lattice pairs $\left(\Lambda_{f}^{(n)}, \Lambda_{c}^{(n)}\right)$ of coding rate $R$ for which

$$
\operatorname{Pr}\left\{\mathbf{Z}_{e q} \notin \mathcal{V}_{f}^{(n)}\right\} \rightarrow 0, \text { as, } n \rightarrow \infty
$$

Proof: The proof follows closely the proof of [12, Theorem 5]. We mention the places where the proof in [12] that have to be modified. Equation (81) in [12] must be modified to take into account that we have two transmitters. Equation [12, (81)] must be modified with Lemma 2. Also Equation [12, (82)] must be modified with Lemma 3. After this, we can continue with the proof in [12] by calculating the Poltyrev exponents and also using the fact that Rogers-good and Poltyrev-good lattices exist. Continuing with these steps in [12] shows that we can obtain the rate of $R<\frac{1}{2} \log \left(\frac{1}{2}+\frac{P}{\sigma^{2}}\right)$, which proves the theorem.

We are ready to present the main theorem in this Section which is given below.

Theorem 5: For the lattice coding scheme described above, any exchange rate $R_{\text {ex,lattice }}<\frac{1}{2} \log \left(\frac{1}{2}+\frac{P}{\sigma^{2}}\right)$ is achievable with lattice (Euclidean) decoding.

Proof:

MAC Phase: We will first show that the probability of error in decoding $\mathbf{t}$ from $\mathbf{y}_{R}$ can be made arbitrarily small for asymptotically large $n$. During the MAC phase, the relay tries to decode to

$$
\mathbf{t}=\left(\mathbf{t}_{1}+\mathbf{t}_{2}\right) \bmod \Lambda
$$


from the received signal $\mathbf{y}_{R}$ (given in $(6)$ as follows. The decoder at the relay node forms $\hat{\mathbf{t}}=\left(\alpha \mathbf{y}_{R}+\mathbf{d}_{1}+\mathbf{d}_{2}\right)$ $\bmod \Lambda_{c}(\alpha$ will be determined later) and finds the lattice point in the fine lattice that is closest to $\hat{\mathbf{t}}$, i.e., the estimate of $\mathbf{t}$ is $Q_{\Lambda_{f}}(\hat{\mathbf{t}})$. Using the distributive property of the mod operation, $\hat{\mathbf{t}}$ can be written as:

$$
\begin{aligned}
\hat{\mathbf{t}}= & \left(\alpha \mathbf{y}+\mathbf{d}_{1}+\mathbf{d}_{2}\right) \bmod \Lambda_{c} \\
= & \left(\alpha\left(\mathbf{x}_{1}+\mathbf{x}_{2}\right)+\alpha \mathbf{z}+\mathbf{d}_{1}+\mathbf{d}_{2}\right) \bmod \Lambda_{c} \\
= & \left(\mathbf{x}_{1}+\mathbf{x}_{2}+\mathbf{d}_{1}+\mathbf{d}_{2}+\alpha \mathbf{z}-(1-\alpha)\left(\mathbf{x}_{1}+\mathbf{x}_{2}\right)\right) \bmod \Lambda_{c} \\
= & \left(\left(\mathbf{t}_{1}-\mathbf{d}_{1}\right) \bmod \Lambda_{c}+\left(\mathbf{t}_{2}-\mathbf{d}_{2}\right) \bmod \Lambda_{c}+\right. \\
& \left.\mathbf{d}_{1}+\mathbf{d}_{2}+\alpha \mathbf{z}-(1-\alpha)\left(\mathbf{x}_{1}+\mathbf{x}_{2}\right)\right) \bmod \Lambda_{c} \\
= & \left(\left(\mathbf{t}_{1}+\mathbf{t}_{2}\right) \bmod \Lambda_{c}+\alpha \mathbf{z}-(1-\alpha)\left(\mathbf{x}_{1}+\mathbf{x}_{2}\right)\right) \bmod \Lambda_{c} \\
= & \left(\mathbf{t}+\alpha \mathbf{z}-(1-\alpha)\left(\mathbf{x}_{1}+\mathbf{x}_{2}\right)\right) \bmod \Lambda_{c}
\end{aligned}
$$

Due to the group structure of the lattice, $\mathbf{t}$ is a lattice point in the fine lattice (more precisely, $\mathbf{t} \in\left\{\Lambda_{f} \cap \mathcal{V}\left(\Lambda_{c}\right)\right\}$ ). From Lemma 1, it can be seen that $\mathbf{t}$ is uniformly distributed over $\mathcal{V}\left(\Lambda_{c}\right)$. Further, note that $\mathbf{t}_{1}$ and $\mathbf{t}_{2}$ are independent of $\mathbf{z}, \mathbf{x}_{1}$ and $\mathbf{x}_{2}$ and, hence, we can define an equivalent noise term as $\mathbf{z}_{e q}=\alpha \mathbf{z}-(1-\alpha)\left(\mathbf{x}_{1}+\mathbf{x}_{2}\right)$ such that $\mathbf{t}$ and $\mathbf{z}_{e q}$ are independent of each other. The second moment of $Z_{e q}$ is given by $\sigma_{e q}^{2}=E\left[Z_{e q}^{2}\right]=\alpha^{2} \sigma^{2}+(1-\alpha)^{2} 2 P$. We can now choose $\alpha$ to minimize $E\left[z_{e q}^{2}\right]$ and the resulting optimum values of $\alpha$ and $\sigma_{e q}^{2}$ are $\alpha_{o p t}=\frac{2 P}{2 P+\sigma^{2}}$ and $\sigma_{\text {eq,opt }}^{2}=\frac{2 P \sigma^{2}}{2 P+\sigma^{2}}$. From Theorem 4, it can be seen that there exist nested lattices of rate $R_{\text {lattice }}<\frac{1}{2} \log \left(\frac{1}{2}+\frac{P}{\sigma^{2}}\right)$ for which $\operatorname{Pr}\left\{\mathbf{Z}_{e q} \notin \mathcal{V}_{f}^{(n)}\right\} \rightarrow 0$, as, $n \rightarrow \infty$. and, hence, the probability of decoding error

$$
P_{e}=\operatorname{Pr}\left\{Q_{\Lambda_{f}}(\hat{\mathbf{t}}) \neq \mathbf{t}\right\} \rightarrow 0 \text { as } n \rightarrow \infty .
$$

Hence, we can use a rate of

$$
R_{\text {Lattice }} \triangleq \frac{1}{n} \log _{2}\left|\Lambda_{f} \cap \mathcal{V}\left(\Lambda_{c}\right)\right|<\frac{1}{2} \log \left(\frac{1}{2}+\frac{P}{\sigma^{2}}\right)
$$

at each of the nodes and $\left(\mathbf{t}_{1}+\mathbf{t}_{2}\right) \bmod \Lambda_{c}$ can be decoded at the relay.

Broadcast Phase:

In the broadcast phase, the relay node transmits the index of $\mathbf{t}$ using a capacity achieving code for the AWGN channel. Since the capacity of the AWGN is $\frac{1}{2} \log \left(1+\frac{P}{\sigma^{2}}\right)$ which is higher than $R_{\text {Lattice }}$ in 17 and, hence, the index (or, equivalently, t) can be obtained at the nodes $A$ and $B$. Since node $A$ already has $\mathbf{u}_{A}$ and, hence, $\mathbf{t}_{1}$, it needs to recover $\mathbf{u}_{B}$ or, equivalently, $\mathbf{t}_{2}$, from $\mathbf{t}$ and $\mathbf{t}_{1}$. This can be done as follows. Node $A$ computes $\left(\mathbf{t}-\mathbf{t}_{1}\right)$ $\bmod \Lambda_{c}$, which can be written as

$$
\begin{aligned}
\left(\mathbf{t}-\mathbf{t}_{\mathbf{1}}\right) \bmod \Lambda_{c} & =\left(\left(\mathbf{t}_{1}+\mathbf{t}_{2}\right) \bmod \Lambda_{c}-\mathbf{t}_{1}\right) \bmod \Lambda_{c} \\
& =\mathbf{t}_{2} \bmod \Lambda_{c} \\
& =\mathbf{t}_{2}
\end{aligned}
$$

Similarly, $\mathbf{t}_{1}$ can also be obtained in node $B$ by taking $\left(\mathbf{t}-\mathbf{t}_{2}\right) \bmod \Lambda_{c}$. Hence, an effective rate of $R_{e x, L a t t i c e}<$ $\frac{1}{2} \log \left(\frac{1}{2}+\frac{P}{\sigma^{2}}\right)$ can be obtained using nested lattices with lattice decoding.

We conclude by noting that, at high SNR, this scheme approaches the upper bound of $\frac{1}{2} \log \left(1+\frac{P}{\sigma^{2}}\right)$ and is therefore nearly optimal.

This scheme can be interpreted as a Slepian-Wolf coding scheme using nested lattices, i.e., the relay wishes to convey $\mathbf{t}_{1}+\mathbf{t}_{2}$ to node $A$, where some side information (namely, $\mathbf{t}_{1}$ ) is available. Thus, the broadcast phase in effect uses nested lattices for solving the Slepian-Wolf coding problem. This scheme can also be thought of as a decode and forward scheme where the relay decodes a function of $\mathbf{t}_{1}$ and $\mathbf{t}_{2}$, namely $\mathbf{t}=\left(\mathbf{t}_{1}+\mathbf{t}_{2}\right) \bmod \Lambda_{c}$ and forwards this to the nodes. Notice however, that the relay will not know either $\mathbf{t}_{1}$ or $\mathbf{t}_{2}$ exactly, it only knows $\mathbf{t}$.

Since the nested lattice code we have used is a capacity achieving code for the AWGN channel, one does not have to encode $\mathbf{t}$ again. The relay can simply transmit $\mathbf{t}$ to the nodes $A$ and $B$ and $\mathbf{t}$ can be decoded at the nodes $A$ and $B$ in the presence of noise at the nodes $A$ and $B$. Notice that $E\left[\| \mathbf{t}||^{2}\right] \leq P$ and, hence, the power constraint will be satisfied at the relay node. 


\section{ViI. Lattice Coding with Minimum Angle Decoding}

In the previous section we observed that nested lattice decoding can achieve an exchange rate of $\frac{1}{2} \log \left(\frac{1}{2}+\frac{P}{\sigma^{2}}\right)$ with lattice decoding alone. This naturally leads us to the question, can we achieve a better performance by using other decoding schemes? In this section we study a suboptimal decoder called the minimum angle decoder [11].

\section{A. Description}

We next briefly explain our minimum angle decoding scheme. We have two transmitters communicating simultaneously to a central router. Both of them have the same power constraint $P$. The noise in the channel is Gaussian having a variance $\sigma^{2}$. As we have seen in previous sections, choosing a good lattice code provides us with a considerable rate gain compared to random codes. Here we consider a $n$-dimensional lattice $\Lambda_{n} \subset \mathbb{R}^{n}$. Let $T_{\sqrt{P}}$ be an $n$-dimensional closed ball, centered at the origin and having a radius $\sqrt{n P}$, and let $V_{n}(\sqrt{n P})$ be the hyper-volume of $T_{\sqrt{P}}$. This can be treated as a power constraint. Our codewords will be composed of lattice points in the sphere $T_{\sqrt{P}}$. Our encoding strategy would be that, each transmitter chooses a lattice point corresponding to its message index and transmits synchronously over the the Gaussian channel. Here we have no nested lattice construction or the use of dither in the encoding stage. At the receiver we will be interested in decoding to the sum of these lattice points.

Minimum Angle Decoder: A minimum angle decoder discussed here makes a decision based on lattice points in a thin $\mathrm{n}$-dimensional spherical shell $T_{\sqrt{2 P}}^{\Delta} \doteq\left\{\mathbf{x} \in \mathbb{R}^{n}: \sqrt{n(2 P-\delta)} \leq\|\mathbf{x}\| \leq \sqrt{n(2 P+\delta)}\right\}, \delta$ is small and non-zero. It takes the received vector and finds the lattice point, whose projection on the thin shell, is closest to the received vector.

An optimal decoder can be seen to be a maximum likelihood decoder. However, it is very tough to analyze this decoder. Hence, we analyze the minimum angle decoder, which is more tractable analytically. We next state the main theorem of this section,

\section{B. Achievable rate}

Theorem 6: For the bi-directional relaying problem considered in Section $\mathbb{1}$, there exists at least one $n$-dimensional lattice $\Lambda_{n}$ such that an exchange rate of $R_{e x}<\frac{1}{2} \log \left(\frac{1}{2}+S N R\right)$ is achievable using a minimum angle decoder as $n \rightarrow \infty$.

\section{Proof Sketch}

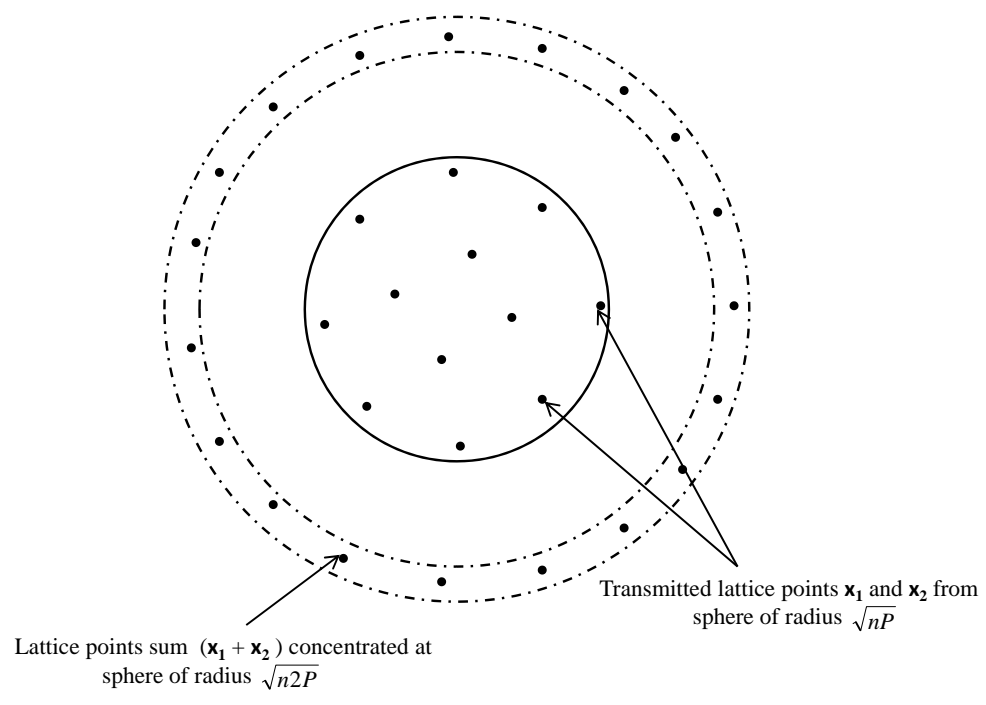

Fig. 3. Picture showing the concentration of the sum of lattice points on the thin shell of radius $\sqrt{n 2 P}$. 
It is well known that the volume of an $n$-dimensional sphere is concentrated mainly on the surface of the sphere as the dimension becomes large. It is also known that, if we intersect a lattice with a $n$-dimensional sphere, then most of the lattice points will be concentrated very close to the surface [11]. In the course of our proof, we will show that the sum of any two such randomly chosen lattice points is also concentrated on a thin spherical shell $T_{\sqrt{2 P}}^{\Delta}$ at a radius of $\sqrt{2 n P}$. Hence, the probability of error will be largely depended on the lattice points in the thin spherical shell $T_{\sqrt{2 P}}^{\Delta}$. We will be using the Blichfeldt's principle to show that there exist translations (one for each user) of the lattice $\Lambda_{n}$ where the sum points are concentrated (see Theorem 9. Lemma 12 and Lemma 13). Once concentration for the sum of lattice points can be established, we can perform minimum angle decoding. In minimum angle decoding, we are interested only in the angle between the different lattice points on the thin spherical shell. It must be noted that the choice of the lattice $\Lambda_{n}$ must be such that it must act as a good channel code. The Minkowski-Hlawka theorem (Theorem 11 and Lemma 14) can be used to show existence of such lattices. Choosing volume of the lattice's Voronoi region appropriately allows us to compute an achievable rate of this scheme.

\section{Detailed proof}

First let us provide some definitions and notation,

- $\Lambda_{n}$ denotes an $n$-dimensional lattice and let $P_{n}$ be the basic Voronoi region of the lattice.

- $\mathbf{s}_{\mathbf{1}}, \mathbf{s}_{\mathbf{2}}$ are two $n$-dimensional translational vectors, $\mathbf{s}_{\mathbf{1}}, \mathbf{s}_{\mathbf{2}} \in P_{n}$.

- $\mathcal{L}(\mathbf{s}, T)$ is defined as the set of lattice points translated by a vector $\mathbf{s}$ present in the region $T$ or otherwise, $\mathcal{L}(\mathbf{s}, T)=\left(\Lambda_{n}+\mathbf{s}\right) \cap T$.

- $T_{\sqrt{P}}$ and $T_{\sqrt{2 P}}$ represents $n$-dimensional hyper-spheres centered at the origin and having a radius of $\sqrt{n P}$ and $\sqrt{n 2 P}$ respectively.

- $T_{\sqrt{2 P}}^{\Delta}$ represents $n$-dimensional thin spherical shell around the radius $\sqrt{n 2 P}$ and is defined as $T_{\sqrt{2 P}}^{\Delta}=\{\mathbf{x} \in$ $\left.\mathbb{R}^{n}: \sqrt{n(2 P-\delta)} \leq\|\mathbf{x}\| \leq \sqrt{n(2 P+\delta)}\right\}, \delta$ is small and non-zero.

- $\mathcal{C}_{1}$ and $\mathcal{C}_{2}$ are codewords formed by intersection of lattice points of hyper-spheres and are given by, $\mathcal{C}_{1}=$ $\mathcal{L}\left(\mathbf{s}_{1}, T_{\sqrt{P}}\right)=\left(\Lambda_{n}+\mathbf{s}_{1}\right) \cap T_{\sqrt{P}}$ and $\mathcal{C}_{2}=\mathcal{L}\left(\mathbf{s}_{\mathbf{2}}, T_{\sqrt{P}}\right)=\left(\Lambda_{n}+\mathbf{s}_{2}\right) \cap T_{\sqrt{P}}$.

- Similarly $\mathcal{C}_{\sqrt{2 P}}$ is given as $\mathcal{C}_{\sqrt{2 P}}=\mathcal{L}\left(\mathbf{s}_{\mathbf{1}}+\mathbf{s}_{\mathbf{2}}, T_{\sqrt{2 P}}\right)=\left(\Lambda_{n}+\mathbf{s}_{1}+\mathbf{s}_{2}\right) \cap T_{\sqrt{2 P}}$ and $\mathcal{C}_{\sqrt{2 P}}^{\Delta}$ is given by $\mathcal{C}_{\sqrt{2 P}}^{\Delta}=\mathcal{L}\left(\mathbf{s}_{1}+\mathbf{s}_{2}, T_{\sqrt{2 P}}^{\Delta}\right)=\left(\Lambda_{n}+\mathbf{s}_{1}+\mathbf{s}_{2}\right) \cap T_{\sqrt{2 P}}^{\Delta}$.

- $\mathcal{C}_{2}$ are codewords formed by intersection of lattice points of hyper-spheres and are given by, $\mathcal{C}_{1}=\mathcal{L}\left(\mathbf{s}_{1}, T_{\sqrt{P}}\right)=$ $\left(\Lambda_{n}+\mathbf{s}_{1}\right) \cap T_{\sqrt{P}}$ and $\mathcal{C}_{2}=\mathcal{L}\left(\mathbf{s}_{\mathbf{2}}, T_{\sqrt{P}}\right)=\left(\Lambda_{n}+\mathbf{s}_{2}\right) \cap T_{\sqrt{P}}$.

- Let $\mathcal{C}_{\oplus}$ be defined as $\mathcal{C}_{\oplus}=\mathcal{C}_{1} \times \mathcal{C}_{2}=\left\{\left(\mathbf{x}_{\mathbf{1}}, \mathbf{x}_{\mathbf{2}}\right): \mathbf{x}_{\mathbf{1}} \in \mathcal{C}_{1}, \mathbf{x}_{\mathbf{2}} \in \mathcal{C}_{2}\right\}$. This denotes the combined collection of pairs of codewords of both the transmitters.

- Again let $\mathcal{C}_{\oplus}^{\Delta}=\left\{\left(\mathbf{x}_{1}, \mathbf{x}_{\mathbf{2}}\right): \mathbf{x}_{\mathbf{1}}+\mathbf{x}_{\mathbf{2}} \in T_{\sqrt{2 P}}^{\Delta}, \mathbf{x}_{\mathbf{1}} \in \mathcal{C}_{1}, \mathbf{x}_{\mathbf{2}} \in \mathcal{C}_{2}\right\}$ This denotes the codeword pairs whose sum lies on the thin shell $T_{\sqrt{2 P}}^{\Delta}$.

- Let $\mathcal{C}_{\oplus}^{\prime}=\mathcal{C}_{\oplus} \backslash \mathcal{C}_{\oplus}^{\Delta}$. This denotes the code word pairs whose sum does not lie on the thin shell. It must be noted that the set formed by the sum of codewords in $\mathcal{C}_{\oplus}^{\Delta}$ need not be the same as $\mathcal{C}_{\sqrt{2 P}}^{\Delta}$ and at low SNR this may lead to significant difference between ML and minimum angle decoding.

- Let $M_{1}, M_{2}, M_{\oplus}, M_{\oplus}^{\Delta}$ and $M_{\oplus}^{\prime}$ denote the cardinality of $\mathcal{C}_{1}, \mathcal{C}_{2}, \mathcal{C}_{\oplus}, \mathcal{C}_{\oplus}^{\Delta}$ and $\mathcal{C}_{\oplus}^{\prime}$ respectively.

- For a given code $\mathcal{C}$, let us denote the average probability of error, under minimum distance decoding as $P^{\mathcal{C}}$.

- Let us define a projection function $\pi$. This projects a $n$ dimensional vector onto to an inner sphere of radius $\sqrt{n(2 P-\delta)}$. It is defined as $\pi(\mathbf{x})=(\sqrt{n(2 P-\delta)} /\|\mathbf{x}\|) \mathbf{x}$.

It is easy to see that minimum distance decoding is equivalent to maximum likelihood decoding in the presence of Gaussian noise.

As mentioned before the set of lattice points whose sum lies in the thin spherical shell $T_{\sqrt{2 P}}^{\Delta}$ is much larger than the lattice points whose sum lies outside the spherical region. Hence the average probability of error will not be affected much by these lattice points. This motivates us to express the average probability of error $P^{\mathcal{C}_{\oplus}}$ as a sum of two terms as made more clear in the lemma below[11].

Lemma 7:

Proof:

$$
P^{\mathcal{C}_{\oplus}} \leq \frac{M_{\oplus}^{\prime}}{M_{\oplus}}+\frac{1}{M_{\oplus}} \sum_{\left(\mathbf{x}_{1}, \mathbf{x}_{2}\right) \in \mathcal{C}_{\oplus}^{\Delta}} P^{\mathcal{C} \oplus}\left(\pi\left(\mathbf{x}_{\mathbf{1}}+\mathbf{x}_{\mathbf{2}}\right)\right)
$$


Let the ordered pair $\left(\mathbf{x}_{1}, \mathbf{x}_{2}\right) \in \mathcal{C}_{\oplus}$, denote that $\mathbf{x}_{1} \in \mathcal{C}_{1}$ and $\mathbf{x}_{\mathbf{2}} \in \mathcal{C}_{2}$. We next follow similar steps of the proof as given in [11]. Let $P^{\mathcal{C}_{\oplus}}\left(\mathbf{x}_{1}, \mathbf{x}_{2}\right)=P^{\mathcal{C}_{\oplus}}\left(\mathbf{x}_{1}+\mathbf{x}_{2}\right)$, denote the probability of error in decoding to the sum $\left(\mathbf{x}_{1}+\mathbf{x}_{2}\right)$, for a pair $\left(\mathbf{x}_{1}, \mathbf{x}_{2}\right) \in \mathcal{C}_{\oplus} \cdot \mathbf{x}_{1}$ and $\mathbf{x}_{2}$ are transmitted simultaneously by user 1 and 2 respectively and the router receives the sum $\mathbf{x}_{1}+\mathbf{x}_{\mathbf{2}}$ corrupted with some Gaussian noise. Now let $\tilde{P}^{\mathcal{C}_{\oplus}}$, represent a suboptimum decoder which maps the received point to the nearest sum $\mathbf{x}_{1}+\mathbf{x}_{2}$, where $\left(\mathbf{x}_{1}, \mathbf{x}_{2}\right) \in \mathcal{C} \Delta$. Hence we can bound the average probability of error as follows

$$
\begin{aligned}
P^{\mathcal{C}_{\oplus}} & \leq \tilde{P}^{\mathcal{C}_{\oplus}} \\
& =\frac{1}{M_{\oplus}} \sum_{\left(\mathbf{x}_{\mathbf{1}}, \mathbf{x}_{\mathbf{2}}\right) \in \mathcal{C}_{\oplus}^{\prime}} \tilde{P}^{\mathcal{C}_{\oplus}}\left(\mathbf{x}_{\mathbf{1}}, \mathbf{x}_{\mathbf{2}}\right)+\frac{1}{M_{\oplus}} \sum_{\left(\mathbf{x}_{\mathbf{1}}, \mathbf{x}_{\mathbf{2}}\right) \in \mathcal{C}_{\oplus}} \tilde{P}^{\mathcal{C}_{\oplus}}\left(\mathbf{x}_{\mathbf{1}}, \mathbf{x}_{\mathbf{2}}\right) \\
& \stackrel{(a)}{=} \frac{M_{\oplus}^{\prime}}{M_{\oplus}}+\frac{1}{M_{\oplus}} \sum_{\left(\mathbf{x}_{\mathbf{1}}, \mathbf{x}_{\mathbf{2}}\right) \in \mathcal{C}_{\oplus}} \tilde{P}^{\mathcal{C}_{\oplus}}\left(\mathbf{x}_{\mathbf{1}}, \mathbf{x}_{\mathbf{2}}\right) \\
& =\frac{M_{\oplus}^{\prime}}{M_{\oplus}}+\frac{1}{M_{\oplus}} \sum_{\left(\mathbf{x}_{\mathbf{1}}, \mathbf{x}_{\mathbf{2}}\right) \in \mathcal{C}_{\oplus}^{\Delta}} \tilde{P}^{\mathcal{C}_{\oplus}}\left(\mathbf{x}_{\mathbf{1}}+\mathbf{x}_{\mathbf{2}}\right) \\
& \stackrel{(b)}{\leq} \frac{M_{\oplus}^{\prime}}{M_{\oplus}}+\frac{1}{M_{\oplus}} \sum_{\left(\mathbf{x}_{\mathbf{1}}, \mathbf{x}_{\mathbf{2}}\right) \in \mathcal{C}_{\oplus}^{\Delta}} P^{\pi\left(\mathcal{C}_{\oplus}^{\Delta}\right)}\left(\mathbf{x}_{\mathbf{1}}+\mathbf{x}_{\mathbf{2}}\right)
\end{aligned}
$$

Here in $(a)$ the first term follows since $M_{\oplus}^{\prime}$ is the cardinality of $\mathcal{C}_{\oplus}^{\prime}$ and the probability of error using our suboptimal decoder is 1 , when $\left(\mathbf{x}_{1}, \mathbf{x}_{2}\right) \in \mathcal{C}_{\oplus}^{\prime}$. In (b) $\pi$ refers to the projection function defined before and $P^{\pi\left(\mathcal{C}_{\oplus}^{\Delta}\right)}$, represents the minimum angle decoder described before. The inequality can be shown to be true by referring to the discussions on [11, Lemma 2].

Next since we are interested in lattice points projected on to the inner sphere of radius $\sqrt{n(2 P-\delta)}$, we can define a decoding algorithm that looks at the angle between the lattice points, the minimum angle decoder. We next establish some more definitions. Let $B_{\theta}(\mathbf{y})$ denote a n-dimensional cone centered at the origin and having the axis passing through $\mathbf{y}$. Let $\theta$ be the half-angle of the cone and $\mathbf{y} \in \mathbb{R}^{n}$ be non-zero.

We next define a sub-optimum decoding function given as follows,

$$
A_{\theta}\left(\mathbf{x}_{\mathbf{1}}, \mathbf{x}_{\mathbf{2}}\right)=B_{\theta}\left(\mathbf{x}_{\mathbf{1}}+\mathbf{x}_{\mathbf{2}}\right) \backslash \underset{\mathbf{x}^{\prime} \in \mathcal{C}_{\sqrt{2 P}}^{\Delta} \backslash\left\{\mathbf{x}_{1}+\mathbf{x}_{\mathbf{2}}\right\}}{\bigcup} B_{\theta}\left(\mathbf{x}^{\prime}\right)
$$

or this can also be expressed as

$$
A_{\theta}^{C}\left(\mathbf{x}_{\mathbf{1}}, \mathbf{x}_{\mathbf{2}}\right)=B_{\theta}^{C}\left(\mathbf{x}_{\mathbf{1}}+\mathbf{x}_{\mathbf{2}}\right) \bigcup_{\mathbf{x}^{\prime} \in \mathcal{C}_{\sqrt{2 P}}^{\Delta} \backslash\left\{\mathbf{x}_{1}+\mathbf{x}_{2}\right\}} B_{\theta}\left(\mathbf{x}^{\prime}\right)
$$

$A_{\theta}\left(\mathbf{x}_{1}, \mathbf{x}_{\mathbf{2}}\right)$ represents the region of the cone $B_{\theta}\left(\mathbf{x}_{\mathbf{1}}+\mathbf{x}_{\mathbf{2}}\right)$, that does not intersect with any other cone corresponding to the other lattice codeword points $\mathbf{x}^{\prime}$, located in the thin spherical shell. During decoding, when we receive a vector that falls in the region $A_{\theta}\left(\mathbf{x}_{1}, \mathbf{x}_{2}\right)$, we decode to the sum codeword $\left(\mathbf{x}_{1}+\mathbf{x}_{2}\right)$. It may not be possible to decode to the individual codewords $\mathbf{x}_{1}$ and $\mathbf{x}_{2}$, as different pairs of codewords may yield the same sum. However it must be noted, that in the forward phase, we are interested in decoding only to the sum of the transmitted codewords. 
Let $P_{\theta}$ denote the probability of error using the sub-optimum decoder. Then, we have

$$
\begin{aligned}
P_{\theta}^{\pi\left(\mathcal{C}_{\oplus}^{\Delta}\right)}\left(\mathbf{x}_{\mathbf{1}}, \mathbf{x}_{\mathbf{2}}\right) & =\operatorname{Pr}\left(\pi\left(\mathbf{x}_{\mathbf{1}}+\mathbf{x}_{\mathbf{2}}\right)+Z^{n} \in A_{\theta}^{C}\left(\mathbf{x}_{\mathbf{1}}, \mathbf{x}_{\mathbf{2}}\right)\right) \\
& \stackrel{(a)}{\leq} \operatorname{Pr}\left(\pi\left(\mathbf{x}_{\mathbf{1}}+\mathbf{x}_{\mathbf{2}}\right)+Z^{n} \notin B_{\theta}\left(\mathbf{x}_{\mathbf{1}}+\mathbf{x}_{\mathbf{2}}\right)\right)+\sum_{\mathbf{x}^{\prime} \in \mathcal{C}_{\sqrt{2 P}}^{\Delta} \backslash\left\{\mathbf{x}_{\mathbf{1}}+\mathbf{x}_{\mathbf{2}}\right\}} \operatorname{Pr}\left(\pi\left(\mathbf{x}_{\mathbf{1}}+\mathbf{x}_{\mathbf{2}}\right)+Z^{n} \in B_{\theta}\left(\mathbf{x}^{\prime}\right)\right) \\
& \stackrel{(b)}{=} \operatorname{Pr}\left(\mathbf{t}_{\mathbf{0}}+Z^{n} \notin B_{\theta}\left(\mathbf{t}_{\mathbf{0}}\right)\right)+\sum_{\mathbf{x}^{\prime} \in \mathcal{C}_{\sqrt{2 P}}^{\Delta} \backslash\left\{\mathbf{x}_{\mathbf{1}}+\mathbf{x}_{\mathbf{2}}\right\}} p_{\theta}\left(\mathbf{x}_{\mathbf{1}}+\mathbf{x}_{\mathbf{2}}, \mathbf{x}^{\prime}\right) \\
& \stackrel{(c)}{=} \operatorname{Pr}\left(\mathbf{t}_{\mathbf{0}}+Z^{n} \notin B_{\theta}\left(\mathbf{t}_{\mathbf{0}}\right)\right)+\sum_{g \in \Lambda_{n} \backslash\{0\}} p_{\theta}\left(\mathbf{x}_{\mathbf{1}}+\mathbf{x}_{\mathbf{2}}, g+\mathbf{x}_{\mathbf{1}}+\mathbf{x}_{\mathbf{2}}\right) \chi_{T_{\sqrt{2 P}}^{\Delta}}\left(g+\mathbf{x}_{\mathbf{1}}+\mathbf{x}_{\mathbf{2}}\right)
\end{aligned}
$$

In the above (a) follows because we use the union bound. In (b), the first term follows, because due to symmetry, the probability is not dependent on the particular $\mathbf{x}_{\mathbf{1}}+\mathbf{x}_{\mathbf{2}}$. The second term follows as we define $p_{\theta}\left(\mathbf{x}, \mathbf{x}^{\prime}\right)$ as $p_{\theta}\left(\mathbf{x}, \mathbf{x}^{\prime}\right)=\operatorname{Pr}\left(\pi(\mathbf{x})+Z^{n} \notin B_{\theta}\left(\mathbf{x}^{\prime}\right)\right)$. In (c), we replace $\mathbf{x}^{\prime}$, by $g+\mathbf{x}_{\mathbf{1}}+\mathbf{x}_{\mathbf{2}}$ and the characteristic function $\chi_{T_{\sqrt{2 P}}^{\Delta}}$, corresponds to lattice points on the thin shell at radius $\sqrt{n 2 P}$.

Hence the average probability of error can be bounded as

$$
\begin{aligned}
P^{\mathcal{C}_{\oplus}} \leq & \operatorname{Pr}\left(\mathbf{t}_{\mathbf{0}}+Z^{n} \notin B_{\theta}\left(\mathbf{t}_{\mathbf{0}}\right)\right)+\frac{M_{\oplus}^{\prime}}{M_{\oplus}}+ \\
& \frac{1}{M_{\oplus}} \sum_{\left(\mathbf{x}_{1}, \mathbf{x}_{\mathbf{2}}\right) \in \mathcal{C}_{\oplus}} \sum_{g \in \Lambda_{n} \backslash\{0\}} p_{\theta}\left(\mathbf{x}_{\mathbf{1}}+\mathbf{x}_{\mathbf{2}}, g+\mathbf{x}_{\mathbf{1}}+\mathbf{x}_{\mathbf{2}}\right) \chi_{T_{\sqrt{2 P}}^{\Delta}}\left(g+\mathbf{x}_{\mathbf{1}}+\mathbf{x}_{\mathbf{2}}\right)
\end{aligned}
$$

The rest of the proof deals with bounding each of the three terms in the above equation by an arbitrarily small quantity, to make the probability of error tend to zero as $n \rightarrow \infty$. Below we briefly explain the requirements.

- The first term can be made very small by choosing the angle $\theta$ appropriately. In effect, we need the noise $Z^{n}$ to be contained inside the cone $B_{\theta}\left(\mathbf{t}_{\mathbf{0}}\right)$ with high probability as the dimension $n$ becomes large.

- For the second term we need the number of codeword pairs whose sum of codewords lies outside the thin spherical shell must be shown to be much lesser than the total number of codeword pairs. In other words we need to show that the sum of lattice points are concentrated in the thin spherical shell around the radius $\sqrt{n 2 P}$. This is shown in Lemma 12 .

- The third term has a summation which is difficult to evaluate and hence we bound it by an integral and evaluate the resulting integral.

- Finally, we require that the number of codewords in each of the inner sphere must be sufficiently large to achieve rates close to $\frac{1}{2} \log \left(\frac{1}{2}+S N R\right)$.

The Blichfeldt's principle(see Theorem 9) can be applied to show concentration of codeword pairs. Lemma 13 in Appendix C, is an application of the Blichfeldt's principle that guarantees that for any given lattice, we can find translations that satisfy $\frac{M_{\oplus}^{\prime}}{M_{\oplus}} \leq 4 \frac{V_{\oplus}^{\prime}}{V_{\oplus}}$. Also it makes sure that we can find enough codewords in the hyper spheres of radius $\sqrt{n P}$, such that we can achieve a rate of $\frac{1}{2} \log \left(\frac{1}{2}+S N R\right)$.

The Minkowski-Hlawka theorem (see Theorem 11 in Appendix A) is used to establish the existence of at least one lattice such that the summation of the third term can be bounded by an integral. This theorem along with Lemma 13 in Appendix C, are used together in Lemma 8 to obtain bounds on both the second and third term. Hence we can effectively rewrite (19) by using these bounds to get,

$$
\begin{aligned}
P^{\mathcal{C}_{\oplus}} & \leq 4 \frac{V_{\oplus}^{\prime}}{V_{\oplus}^{\Delta}}+\operatorname{Pr}\left(\mathbf{t}_{\mathbf{0}}+Z^{n} \notin B_{\theta}\left(\mathbf{t}_{\mathbf{0}}\right)\right) \\
& +\left[2 \frac{(n-1) \pi^{\frac{n-1}{2}}(n(2 P+\delta))^{n / 2}}{d_{n} n \Gamma\left(\frac{n+1}{2}\right)} \int_{0}^{\theta} \sin ^{n-2}(x) d x\right]
\end{aligned}
$$

We can bound the integral, as shown in [18, p. 623-624] to get, 


$$
P^{\mathcal{C}_{\oplus}} \leq \operatorname{Pr}\left(\mathbf{t}_{\mathbf{0}}+Z^{n} \notin B_{\theta}\left(\mathbf{t}_{\mathbf{0}}\right)\right)+4 \frac{V_{\oplus}^{\prime}}{V_{\oplus}^{\Delta}}+\left[2 \frac{\pi^{\frac{n-1}{2}}(n(2 P+\delta))^{n / 2}}{d_{n} n \Gamma\left(\frac{n+1}{2}\right)} \frac{\sin ^{n-1}(\theta)}{\cos \theta}\right]
$$

Now we next need to choose the appropriate values for $\theta$ and $d_{n}$ to make the probabilities go to 0 . For the second term, consider $\sin \angle\left(t_{0}+Z, t_{0}\right)$ which is given by

$$
\sin \angle\left(t_{0}+Z, t_{0}\right)=\sqrt{\frac{\left\|Z^{\perp}\right\|^{2}}{\left(\sqrt{n(2 P-\delta)}+Z^{\|}\right)^{2}+\left\|Z^{\perp}\right\|^{2}}} \stackrel{n \rightarrow \infty}{\longrightarrow} \sqrt{\frac{\sigma^{2}}{2 P-\delta+\sigma^{2}}}
$$

Hence we choose $\sin \theta=\sqrt{\frac{\sigma^{2}}{2 P-\delta+\sigma^{2}}}$. For the third term a good choice of $d_{n}$ is

$$
d_{n}=V_{n}(\sqrt{n(2 P+2 \delta)}) \sin ^{n} \theta=\frac{\pi^{n / 2}(n(2 P+2 \delta))^{n / 2}\left(\sin ^{n} \theta\right)}{\Gamma(n / 2+1)}
$$

This choice helps us to make the third term tend to 0 for large $n$. The third term then can be rewritten as given below. We use the results in [11, p. 277], to bound the Gamma functions to get,

$$
\begin{array}{r}
{\left[2 \frac{\pi^{\frac{n-1}{2}}(n(2 P+\delta))^{n / 2}}{d_{n} n \Gamma\left(\frac{n+1}{2}\right)} \frac{\sin ^{n-1}(\theta)}{\cos \theta}\right]=\frac{2}{\sqrt{\pi} \sin \theta \cos \theta}\left(\frac{2 P+\delta}{2 P+2 \delta}\right)^{n / 2} \frac{\Gamma\left(\frac{n}{2}+1\right)}{n \Gamma\left(\frac{n+1}{2}\right)}} \\
<\frac{2}{\sqrt{\pi} \sin \theta \cos \theta}\left(\frac{2 P+\delta}{2 P+2 \delta}\right)^{n / 2} \frac{1}{2} \frac{\left[1+\Gamma\left(\frac{n+1}{2}\right)\right]}{\Gamma\left(\frac{n+1}{2}\right)}
\end{array}
$$

This decays to 0 exponentially as $n \rightarrow \infty$.

Now, the achievable rate can be obtained from the number of lattice points in the sphere of radius $\sqrt{n P}$. This value $M\left(\Lambda_{n}, \mathbf{s}_{\mathbf{1}}^{*}\right), M\left(\Lambda_{n}, \mathbf{s}_{\mathbf{2}}^{*}\right)$, from the lemma, can be seen to be greater than $\frac{V}{8 d_{n}}$. Hence the rate $R$ is given by,

$$
\begin{aligned}
R & \geq \frac{1}{n} \log M\left(\Delta_{n}, \mathbf{s}_{\mathbf{1}}^{*}\right) \\
& \geq \frac{1}{n} \log \frac{V}{8 d_{n}} \\
& \geq \frac{1}{n} \log \left[\frac{1}{8}\left(\frac{P}{(2 P+2 \delta) \sin ^{2} \theta}\right)^{n / 2}\right] \\
& \geq \frac{1}{2} \log \left(\frac{P}{2 P+2 \delta}+\left(\frac{2 P-\delta}{2 P+2 \delta}\right) \frac{P}{\sigma^{2}}\right)-\frac{\log 8}{n} \\
& \geq \frac{1}{2} \log \left(\frac{1}{2}+\frac{P}{\sigma^{2}}\right)-\delta^{\prime}
\end{aligned}
$$

Choosing $\delta^{\prime}$ arbitrarily small, a rate of $\frac{1}{2} \log \left(\frac{1}{2}+\frac{P}{\sigma^{2}}\right)$ can be achieved.

\section{Relationship with ML decoder}

There are some conditions under which the the minimal angle decoder will perform like the ML decoder.

(1) It can be easily seen that, for Gaussian noise, the ML decoder is equivalent to minimum distance decoder.

(2) If all the codewords are concentrated (with high probability) in the thin shell then, we do not lose much by neglecting the codewords outside the thin shell.

(3) Suppose the width of the thin shell is very small and almost all the codewords have approximately the same distance from the origin. Then, calculating minimum distance from received vector to the codewords is equivalent to calculating the minimum angle.

(4) Suppose almost all the lattice points on the thin shell are codewords. Then, decoding to any lattice point in the thin shell does not sacrifice performance.

We are dealing with Gaussian noise so the first condition is easily satisfied. In the course of our proof, we will observe by applying the Blichfeldt's principle that there exists a concentration of codewords at the thin shell. Hence 
condition (2) also holds. Moreover, we will also let the width of the thin shell become arbitrarily small, hence what we are doing is very close to ML decoding. The 4th condition appears not hold to at low SNR, however. In this case, all lattice points in the thin shell may not be codewords. Hence, we think this may lead to the sub-optimality at low SNR. The theorem shows that, for $\mathrm{SNR}<1 / 2$, we get zero rate. We know that, for random Gaussian codebooks, joint decoding of both codewords is possible though. Therefore, we think that the ML decoder may give a better performance at low SNR.

\section{JoInt DECODING BASED SCHEME}

While the aforementioned scheme is nearly optimal at high SNR, the performance of this scheme at low SNR is very poor. In fact, for $P / \sigma^{2}<1 / 2$, the scheme does not even provide a non-zero rate. In this regime, we can use any coding scheme which is optimal for the multiple access channel and perform joint decoding at the relay such that $\mathbf{u}_{A}$ and $\mathbf{u}_{B}$ can be decoded. Then, the relay can encode $\mathbf{u}_{A} \oplus \mathbf{u}_{B}$ and transmit to the nodes. Any coding scheme that is optimal for the MAC channel can be used in the MAC phase. A simple scheme is time sharing (although it is not the only one) where nodes $A$ and $B$ transmit with powers $2 P$ for a duration of $n / 2$ channel uses each but they do not interfere with one another. In this case, a rate of

$$
R_{J D}=\frac{1}{4} \log \left(1+\frac{2 P}{\sigma^{2}}\right)
$$

can be obtained. It can be seen that this is optimal at asymptotically low $\mathrm{SNR}$, since $\log (1+\mathrm{snr}) \approx \mathrm{snr}$ for snr $\rightarrow 0$.

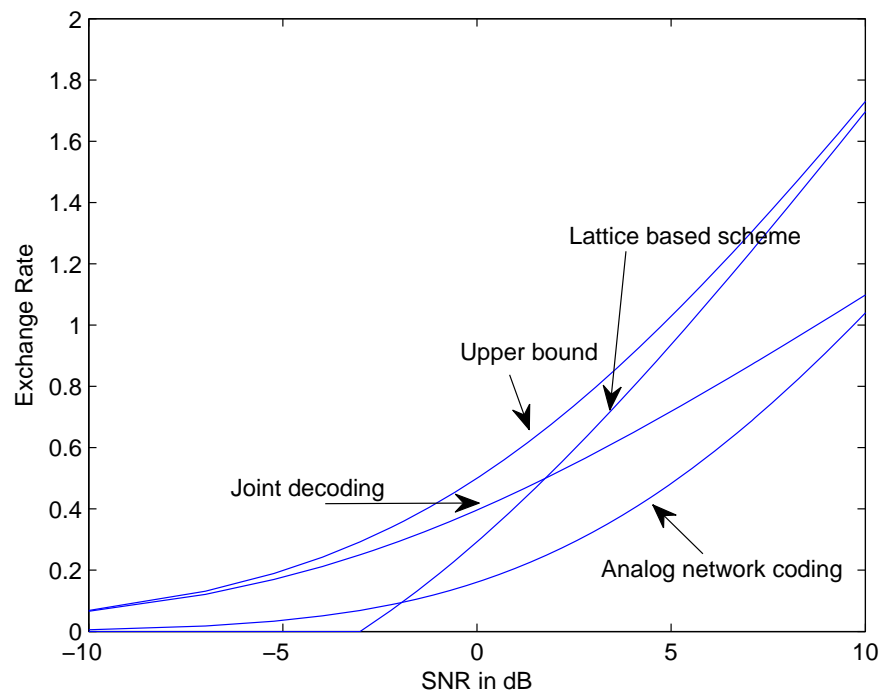

Fig. 4. Achievable Exchange Rates for the Proposed Schemes

The performance of these schemes is shown in Fig. 4 where the upper bound and the achievable rates with these proposed schemes are shown. The achievable rate with the analog network coding scheme is also shown. It can be seen that our schemes outperform analog network coding. However, it must be noted here that the scheme proposed here requires perfect synchronization of the phases from the transmissions, whereas the analog network coding scheme does not.

Clearly, we can time share between the lattice based scheme and the joint decoding based scheme in order to obtain rates of the form

$$
R_{e x}=\beta R_{e x, J D}+(1-\beta) R_{e x, \text { Lattice }} .
$$

It can be shown that between the SNRs of $-0.659 \mathrm{~dB}$ and $3.46 \mathrm{~dB}$, time sharing between the two schemes results in better rates than the individual schemes. In both the lattice based scheme and the joint decoding based scheme, 
if the restriction to use $n$ channel uses during the MAC phase and $n$ during the broadcast phase is removed, i.e., only the total number of uses is constrained to be $2 n$, better schemes can be easily designed. Similarly, different power sharing may also lead to better schemes.

\section{EXTENSION TO MULTIPLE HOPS}

\section{A. Description}

We can extend the above results to multiple hops. We can again show that rate of $\frac{1}{2} \log \left(\frac{1}{2}+\frac{P}{\sigma^{2}}\right)$ is achievable using structured coding even in the multiple hop scenario. It should be noted that the advantage of this scheme over the amplify and forward scheme [3] becomes more pronounced in the multi-hop case, since at each stage for the amplify and forward scheme, the channel noise is amplified and hence the amplify and forward scheme will suffer a huge rate loss as the number of hops increase. The problem model is shown in Fig. 5. The relay nodes and the nodes $A$ and $B$ can transmit only to the two nearest nodes. During a single transmission slot ( $n$ uses of the channel), a node can either listen or transmit. That is, it can not do both simultaneously. We explain our structured coding scheme using a simple example of a 3-relay network. The different transmissions are shown in the table given below.

Here node $A$ and node $B$ have data that need to be exchanged between each other. Each node has a stream of packets. Node $A$ has packets named $\mathbf{u}_{A, 1}, \mathbf{u}_{A, 2}, \ldots$ and node $B$ has packets named $\mathbf{u}_{B, 1}, \mathbf{u}_{B, 2}, \ldots$ In the first transmission slot the nodes $A$ and $B$ transmit. Nodes $A, B$ transmit vectors $\mathbf{x}_{A, 1}$ and $\mathbf{x}_{B, 1}$, respectively using our proposed lattice coding scheme. At the beginning of transmission, the node $R_{2}$ has no data to transmit in the first transmission slot, and hence it remains silent. The node $R_{1}$ and $R_{3}$ decode to $\mathbf{x}_{1,1} \bmod \Lambda$ and $\mathbf{x}_{2,1} \bmod \Lambda$, respectively. During the second transmission slot the nodes $R_{1}$ and $R_{3}$ transmit, while the other nodes remain silent. So, in each stage the nodes transmit and the listening nodes decode to a lattice point in the fine lattice in the Voronoi region of the coarse lattice. In every second transmission slot a new packet is transmitted to the relay nodes by the nodes $A$ and $B$ as can be seen from Table 1 . During slots 2, 4,6 nodes $A, B$ transmits new packets into the relay channel. From this example, we can see that at the 4 th slot the node $A$ and $B$ decode $\mathbf{x}_{B, 1}$ and $\mathbf{x}_{1,2}$ respectively. This is because the node $A$ receives $\left(\mathbf{x}_{1,1}+\mathbf{x}_{1,2}+\mathbf{x}_{2,1}\right) \bmod \Lambda$ during the 4th transmission and, hence, since $\mathbf{x}_{1,1}, \mathbf{x}_{1,2}$ are already known at the node $A$, the node $A$ can decode to $\mathbf{x}_{2,1}$ using modulo operation. The same argument holds for node $B$. From every two transmissions from this stage a new packet can be decoded at each node. This shows that for sufficiently large number packets we can achieve the rate of $\frac{1}{2} \log \left(\frac{1}{2}+\frac{P}{\sigma^{2}}\right)$. A similar encoding scheme can be used for $L=2$ nodes also, in the first slot, node A and $R_{2}$ transmit, while the others listen. In the next slot $R_{1}$ and node $B$ transmit while the others listen and decode. Again the same rate of $\frac{1}{2} \log \left(\frac{1}{2}+\frac{P}{\sigma^{2}}\right)$ is achievable.

\section{B. Achievable rate}

Theorem 8: For the multi hop scenario defined with $L$ hops, an exchange rate of $\frac{1}{2} \log \left(\frac{1}{2}+\frac{P}{\sigma^{2}}\right)$ is achievable with nested lattice encoding and decoding.

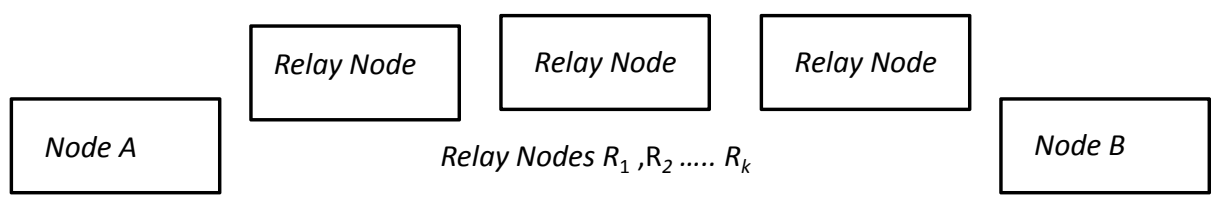

Fig. 5. Problem model for multi hop

Proof: We can easily prove that the theorem holds for a general case $L$ relay nodes in between. In our coding scheme in every two slots a new packet is sent out from the nodes $A$ and $B$. After an initial $2 L$ transmission slot delay, in every two slots the relay nodes receives a new packet from the other nodes. Here, we mean that every two slots the relay node decodes to a lattice point which is a linear function of a new packet. Hence, at the decoding stage at the nodes $B$ and $A$, we can decode after every two slots since only one variable is unknown, since only one new packet (or function of new packet) moves from one node to the other. Hence, we can still achieve the 
TABLE I

Table Showing Sequence of Packets in the Multihop Case

\begin{tabular}{|c|c|c|c|c|c|}
\hline Slot & Node $A$ & Node $R_{1}(\bmod \Lambda)$ & Node $R_{2}(\bmod \Lambda)$ & Node $R_{3}(\bmod \Lambda)$ & Node $B$ \\
\hline 1 & Transmits $\mathbf{x}_{1,1}$ & $\mathbf{x}_{1,1}$ & Transmits & $\mathbf{x}_{2,1}$ & Transmits $\mathbf{x}_{2,1}$ \\
\hline 2 & Remains Silent & Transmits & $\mathbf{x}_{1,1}+\mathbf{x}_{2,1}$ & Transmits & Remains Silent \\
\hline 3 & Transmits $\mathbf{x}_{1,2}$ & $\mathbf{x}_{1,2}+\mathbf{x}_{1,1}+\mathbf{x}_{2,1}$ & Transmits & $\mathbf{x}_{1,1}+\mathbf{x}_{2,1}+\mathbf{x}_{2,2}$ & Transmits $\mathbf{x}_{2,2}$ \\
\hline 4 & Decodes $\mathbf{x}_{2,1}$ & Transmits & $\begin{array}{l}2\left(\mathbf{x}_{1,1}+\mathbf{x}_{2,1}\right)+ \\
\mathbf{x}_{1,2}+\mathbf{x}_{2,2}\end{array}$ & Transmits & Decodes $\mathbf{x}_{1,1}$ \\
\hline 5 & Transmits $\mathbf{x}_{1,3}$ & $\begin{array}{l}2\left(\mathbf{x}_{1,1}+\mathbf{x}_{2,1}\right) \\
+\mathbf{x}_{1,2}+\mathbf{x}_{2,2}+\mathbf{x}_{1,3}\end{array}$ & Transmits & $\begin{array}{l}2\left(\mathbf{x}_{1,1}+\mathbf{x}_{2,1}\right) \\
+\mathbf{x}_{1,2}+\mathbf{x}_{2,2}+\mathbf{x}_{2,3}\end{array}$ & Transmits $\mathbf{x}_{2,3}$ \\
\hline 6 & Decodes $\mathbf{x}_{2,2}$ & Transmits & $\begin{array}{l}4\left(\mathbf{x}_{1,1}+\mathbf{x}_{2,1}\right)+ \\
2\left(\mathbf{x}_{1,2}+\mathbf{x}_{2,2}\right)+ \\
\mathbf{x}_{1,3}+\mathbf{x}_{2,3}\end{array}$ & Transmits & Decodes $\mathbf{x}_{1,2}$ \\
\hline
\end{tabular}

rate of $\frac{1}{2} \log \left(\frac{1}{2}+\frac{P}{\sigma^{2}}\right)$. Moreover, the functions in each stage are bounded for a finite $L$ and, hence, we can always perform the decoding at the receiver nodes.

\section{COnClusion}

We considered joint physical layer and network layer coding for the bi-directional relay problem where two nodes wish to exchange information through AWGN channels. Under the restrictive model of the MAC and broadcast phase using $n$ channel uses separately, we showed upper bounds on the exchange capacity and constructive schemes based on lattices that is nearly optimal at high SNR. At low SNR joint decoding based schemes (optimal coding schemes for the MAC channel) are nearly optimal. These schemes outperform the recently proposed analog network coding. Interestingly, our result shows that structured codes such as lattice codes outperform random codes for such networking problems. We also showed that minimal angle decoding also leads to similar results. We also showed extensions of this scheme to a network with many relay nodes, where the advantages of the proposed scheme over simple amplify and forward will be higher than in single relay case.

\section{APPENDIX A}

\section{BLICHFELDT's PRINCIPLE AND MINKOWSKI-HLAWKA THEOREM}

Theorem 9 (Blichfeldt's Principle [16]): Let $f$ be a Riemann integrable function with bounded support. If $\Lambda_{n}$ is a lattice with fundamental region $P_{n}$ then

Let us define the following function $V_{\oplus}$ as follows,

$$
\int_{\mathbb{R}^{n}} f(\mathbf{s}) d V(\mathbf{s})=\int_{P_{n}}\left(\sum_{h \in \Lambda_{n}} f(h+\mathbf{s})\right) d V(\mathbf{s}) .
$$

$$
V_{\oplus}=\iint \chi_{T}(\mathbf{u}) \chi_{T}(\mathbf{v}) d V(\mathbf{u}) d V(\mathbf{v}) .
$$

Here $V_{\oplus}=\left(V_{n}(\sqrt{n P})\right)^{2}$, represents the square of the volume of an $n$-dimensional sphere of radius $\sqrt{n P} . d V$ represents the $n$-dimensional volume element in rectangular co-ordinates.

We next establish the following corollary

Corollary 10:

Proof: Let us define a function

$$
V_{\oplus}=\int_{\mathbf{s}_{\mathbf{1}}} \int_{\mathbf{s}_{\mathbf{2}}} M_{\oplus}\left(\Lambda_{n}, \mathbf{s}_{\mathbf{1}}, \mathbf{s}_{\mathbf{2}}\right) d V\left(\mathbf{s}_{\mathbf{1}}\right) d V\left(\mathbf{s}_{\mathbf{2}}\right) .
$$

$$
f(\mathbf{u}, \mathbf{v})=\chi_{T}(\mathbf{u}) \chi_{T}(\mathbf{v}) .
$$

For a fixed $\mathbf{u}, f(\mathbf{u}, \mathbf{v})$ can be seen as a function with bounded support and also can be seen to be integrable. Hence we can apply the Blichfeldt's principle to get

$$
h(\mathbf{u})=\int f(\mathbf{u}, \mathbf{v}) d V(\mathbf{v})=\int_{P_{n}}\left(\sum_{h_{2} \in \Lambda_{n}} f\left(\mathbf{u}, h_{2}+\mathbf{s}_{\mathbf{2}}\right)\right) d V\left(\mathbf{s}_{\mathbf{2}}\right) .
$$


Now $h(\mathbf{u})$ can again be seen as a Riemann integrable function with bounded support, and hence the Blichfeldt's principle could be applied again to get the following,

$$
\begin{aligned}
V_{\oplus} & =\int h(\mathbf{u}) d V(\mathbf{u}) \\
& =\int_{P_{n}} \sum_{h_{1} \in \Lambda_{n}}\left(\int_{P_{n}}\left(\sum_{h_{2} \in \Lambda_{n}} f\left(h_{1}+\mathbf{s}_{\mathbf{1}}, h_{2}+\mathbf{s}_{\mathbf{2}}\right)\right) d V\left(\mathbf{s}_{\mathbf{2}}\right)\right) d V\left(\mathbf{s}_{\mathbf{1}}\right) \\
& \stackrel{(a)}{=} \int_{P_{n}} \int_{P_{n}}\left(\sum_{h_{1} \in \Lambda_{n}} \sum_{h_{2} \in \Lambda_{n}} f\left(h_{1}+\mathbf{s}_{\mathbf{1}}, h_{2}+\mathbf{s}_{\mathbf{2}}\right)\right) d V\left(\mathbf{s}_{\mathbf{1}}\right) d V\left(\mathbf{s}_{\mathbf{2}}\right) \\
& \stackrel{(b)}{=} \int_{P_{n}} \int_{P_{n}} M_{\oplus}\left(\Lambda_{n}, \mathbf{s}_{\mathbf{1}}, \mathbf{s}_{\mathbf{2}}\right) d V\left(\mathbf{s}_{\mathbf{1}}\right) d V\left(\mathbf{s}_{\mathbf{2}}\right)
\end{aligned}
$$

Above (a) follows since we have a finite number of non-zero terms, and hence the integral and the summation can be interchanged. Also in (b), $M_{\oplus}\left(\Lambda_{n}, \mathbf{s}_{\mathbf{1}}, \mathbf{s}_{\mathbf{2}}\right)$ is the number of pairs of lattice points for the translations $\mathbf{s}_{\mathbf{1}}$ and $\mathbf{s}_{2}$.

In short the Corollary 8 relates the square of the volume of an $n$-dimensional sphere and number of pairs of lattice points for different translations.

Theorem 11 (Minkowski-Hlawka): Let $f$ be a nonnegative Riemann integrable function with bounded support. Then for every $d \in \mathbb{R}^{+}$and $n \geq 2$, there exists a lattice $\Lambda_{n}$ with determinant $\operatorname{det}\left(\Lambda_{n}\right)=d$ such that

$$
d \sum_{g \in \Lambda_{n} \backslash\{0\}} f(g) \leq \int f d V(\mathbf{x})
$$

The Minkowski-Hlawka theorem gives us a way to connect a series of discrete sums with a continuous integral. This will find applications in our probability of error calculations.

\section{APPENDIX B}

\section{HyPER Volume CONCENTRATION LEMma}

Lemma 12: Let $V_{\oplus}^{\prime}$ be defined as

$$
V_{\oplus}^{\prime}=\iint \chi_{T}(\mathbf{u}) \chi_{T}(\mathbf{v}) \chi_{T_{\sqrt{2 P}}^{\prime}}(\mathbf{u}+\mathbf{v}) d V(\mathbf{u}) d V(\mathbf{v})
$$

then we can choose $n$ sufficiently large such that, $\frac{V_{\oplus}^{\prime}}{V_{\oplus}}<\delta$, for every given positive $\delta$.

Proof:

First we perform a change of variables in the integral, by substituting $\mathbf{x}=\mathbf{u}+\mathbf{v}$. This gives,

$$
V_{\oplus}^{\prime}=\iint \chi_{T}(\mathbf{u}) \chi_{T}(\mathbf{x}-\mathbf{u}) \chi_{T_{\sqrt{2 P}}^{\prime}}(\mathbf{x}) d V(\mathbf{u}) d V(\mathbf{x})
$$

Let us consider first the inner integral, for a fixed $\mathbf{x}$,given by,

$$
\int \chi_{T}(\mathbf{u}) \chi_{T}(\mathbf{x}-\mathbf{u}) d V(\mathbf{u})
$$

This geometrically represents the hyper volume of intersection of two hyper-spheres, whose centers are at a distance $\|\mathbf{x}\|$, from each other. This is pictorially shown in Fig. 6. The calculation of hyper volume of intersection, reduces to obtaining the hyper volume of the conical section and a cone. This is shown pictorially in the second diagram in Fig. 6. Here opq represents the hyper cone and oprq represents the conical section. Here we denote by $V_{c s}(|\mathbf{x}|)$ to represent the volume of the conical section and $V_{c o}(|\mathbf{x}|)$ as the volume of the cone. The integral can hence be be evaluated as

$$
\int_{T} \chi_{T}(\mathbf{v}) \chi_{T}(\mathbf{x}-\mathbf{v}) d \mathbf{v}=2\left(V_{c s}(|\mathbf{x}|)-V_{c o}(|\mathbf{x}|)\right)
$$




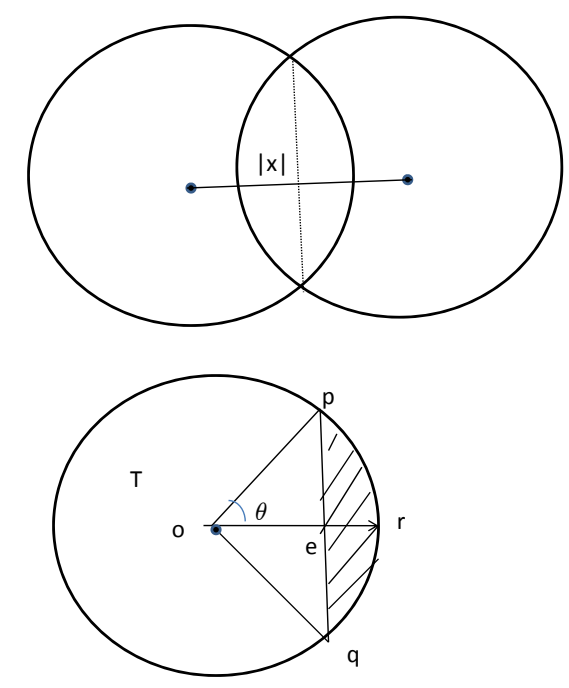

Fig. 6. Geometrical interpretation of the integral

To simplify calculations we can bound the integral as,

$$
\int_{T} \chi_{T}(\mathbf{v}) \chi_{T}(\mathbf{x}-\mathbf{v}) d V(\mathbf{v}) \leq 2 V_{c s}(|\mathbf{x}|)
$$

From the figure we can see $o e=\|x\| / 2$. Hence the half-angle $\theta$ can be calculated as $\cos \theta=\frac{o e}{\sqrt{n P}}=\frac{\|x\|}{2 \sqrt{n P}}$. Hence with the defined $\theta$, we can calculate the hyper volume as,

$$
V_{c s}(|\mathbf{x}|)=2\left(\int_{\psi=0}^{\theta} \sin ^{n-2} \psi d \psi\right) \frac{\pi^{\frac{n-1}{2}}(n P)^{\frac{n}{2}}}{n \Gamma\left(\frac{n-1}{2}\right)}
$$

Hence,

$$
\frac{V_{\oplus}^{\prime}}{V_{\oplus}} \leq 2 \int \frac{V_{c s}(\|\mathbf{x}\|)}{V_{\oplus}} \chi_{T_{\sqrt{2 P}}^{\prime}}(\mathbf{x}) d V(\mathbf{x})
$$

But $V_{\oplus}$ is given by $V^{2}$, where $V$ is the hyper volume of an n-dimensional hyper sphere of radius $\sqrt{n P}$ denoted by $V_{n}(\sqrt{n P})$.

The value of $V_{c s}(\|x\|)$ depends only on the distance of $\mathbf{x}$ from the origin. To make evaluation of the integral easier, we change the volume element to circular co-ordinates and integrate. Thus the integral now becomes,

$$
\frac{V_{\oplus}^{\prime}}{V_{\oplus}} \leq 2\left(\frac{n \pi^{\frac{n}{2}}}{\Gamma\left(\frac{n}{2}+1\right)}\right) \int_{r \in l_{\oplus}^{\prime}} \frac{V_{c s}(r)}{V_{n}(\sqrt{n P})^{2}} r^{n-1} d r
$$

where $l_{\oplus}^{\prime}$ is defined as the union of closed intervals,(will be given later). Now substituting $V_{c s}(r)$ from above gives,

$$
\frac{V_{\oplus}^{\prime}}{V_{\oplus}} \leq 4 \frac{n \pi^{\frac{n}{2}}}{\Gamma\left(\frac{n}{2}+1\right)} \frac{\pi^{\frac{n-1}{2}}(n P)^{\frac{n}{2}}}{n \Gamma\left(\frac{n-1}{2}\right)} \int_{r \in l_{\oplus}^{\prime}} \int_{\psi=0}^{\cos ^{-1}\left(\frac{r}{2 \sqrt{n P}}\right)} \frac{r^{n-1} \sin ^{n-2} \psi}{V_{n}(\sqrt{n P})^{2}} d \psi d r
$$

Now let us choose $\cos \theta=\frac{r}{2 \sqrt{n P}}$. Then change of variables gives

$$
\frac{V_{\oplus}^{\prime}}{V_{\oplus}} \leq 4 \frac{n \pi^{\frac{n}{2}}}{\Gamma\left(\frac{n}{2}+1\right)} \frac{\pi^{\frac{n-1}{2}}(n P)^{\frac{n}{2}}}{n \Gamma\left(\frac{n-1}{2}\right)} \int_{\theta \in \theta_{\oplus}^{\prime}}(n P)^{n / 2} \cos ^{n-1} \theta \sin \theta \int_{\psi=0}^{\theta} \frac{2^{n} \sin ^{n-2} \psi}{V_{n}(\sqrt{n P})^{2}} d \psi d \theta
$$


Substituting for $V_{n}(\sqrt{n P})$, we get,

$$
\begin{aligned}
\frac{V_{\oplus}^{\prime}}{V_{\oplus}} \leq \frac{4}{\sqrt{\pi}} \frac{\Gamma\left(\frac{n}{2}+1\right)}{\Gamma\left(\frac{n-1}{2}\right)} \int_{\theta \in \theta_{\oplus}^{\prime}} 2^{n} \cos ^{n-1} \theta \sin \theta \int_{\psi=0}^{\theta} \sin ^{n-2} \psi d \psi d \theta \\
\frac{V_{\oplus}^{\prime}}{V_{\oplus}} \leq \frac{4}{\sqrt{\pi}} \frac{\Gamma\left(\frac{n}{2}+1\right)}{\Gamma\left(\frac{n-1}{2}\right)} \int_{\theta \in \theta_{\oplus}^{\prime} \cap\left[0, \frac{\pi}{3}+\eta\right]} 2^{n} \cos ^{n-1} \theta \sin \theta \int_{\psi=0}^{\theta} \sin ^{n-2} \psi d \psi d \theta \\
+\frac{4}{\sqrt{\pi}} \frac{\Gamma\left(\frac{n}{2}+1\right)}{\Gamma\left(\frac{n-1}{2}\right)} \int_{\theta \in \theta_{\oplus}^{\prime} \cap\left[\frac{\pi}{3}+\eta, \frac{\pi}{2}\right]} 2^{n} \cos ^{n-1} \theta \sin \theta \int_{\psi=0}^{\theta} \sin ^{n-2} \psi d \psi d \theta
\end{aligned}
$$

Now we will use the bound by Shannon for the first term. We can apply the bound for $\theta<\pi / 2$. Hence we split the integral into two terms to apply the bound. For the second term we will bound $\sin \psi$ by 1 . This gives,

$$
\begin{aligned}
\frac{V_{\oplus}^{\prime}}{V_{\oplus}} \leq & \frac{4}{\sqrt{\pi}} \frac{\Gamma\left(\frac{n}{2}+1\right)}{\Gamma\left(\frac{n-1}{2}\right)} \int_{\theta \in \theta_{\oplus}^{\prime} \cap\left[0, \frac{\pi}{3}+\eta\right]} 2^{n} \cos ^{n-1} \theta \sin \theta \frac{\sin ^{n-1} \theta}{(n-1) \cos \theta} d \theta \\
& +\frac{4}{\sqrt{\pi}} \frac{\Gamma\left(\frac{n}{2}+1\right)}{\Gamma\left(\frac{n-1}{2}\right)} \int_{\theta \in \theta_{\oplus}^{\prime} \cap\left[\frac{\pi}{3}+\eta, \frac{\pi}{2}\right]} 2^{n} \cos ^{n-1} \theta \sin \theta \int_{\psi=0}^{\theta} d \psi d \theta
\end{aligned}
$$

Simplifying things further we get,

$$
\begin{aligned}
\frac{V_{\oplus}^{\prime}}{V_{\oplus}} & \leq \frac{4}{\sqrt{\pi}} \frac{\Gamma\left(\frac{n}{2}+1\right)}{\Gamma\left(\frac{n+1}{2}\right)} \int_{\theta \in \theta_{\oplus}^{\prime} \cap\left[0, \frac{\pi}{3}+\eta\right]} 2^{n-1} \sin ^{n-1} \theta \cos ^{n-1} \theta \tan \theta d \theta \\
& +\frac{4}{\sqrt{\pi}} \frac{(n-1) \Gamma\left(\frac{n}{2}+1\right)}{\Gamma\left(\frac{n+1}{2}\right)} \int_{\theta \in \theta_{\oplus}^{\prime} \cap\left[\frac{\pi}{3}+\eta, \frac{\pi}{2}\right]} 2^{n-1} \cos ^{n-1} \theta \sin \theta \frac{\pi}{2} d \theta
\end{aligned}
$$

Next we bound $\tan \theta$ in the first term by $\tan (\pi / 3+\eta)$ and $\sin \theta$ in the second term by 1 . We also bound the factorial using the bound given in Urbanke. This yields,

$$
\begin{aligned}
\frac{V_{\oplus}^{\prime}}{V_{\oplus}} \leq & \frac{4}{\sqrt{\pi}} \frac{\frac{n}{2}\left(1+\Gamma\left(\frac{n+1}{2}\right)\right) \tan \left(\frac{\pi}{3}+\eta\right)}{\Gamma\left(\frac{n+1}{2}\right)} \int_{\theta \in \theta_{\oplus}^{\prime} \cap\left[0, \frac{\pi}{3}+\eta\right]}(\sin 2 \theta)^{n-1} d \theta \\
& +\frac{4}{\sqrt{\pi}} \frac{(n-1) \frac{n}{2}\left(1+\Gamma\left(\frac{n+1}{2}\right)\right)}{\Gamma\left(\frac{n+1}{2}\right)} \int_{\theta \in \theta_{\oplus}^{\prime} \cap\left[\frac{\pi}{3}+\eta, \frac{\pi}{2}\right]} \frac{\pi}{2}(2 \cos \theta)^{n-1} d \theta
\end{aligned}
$$

Again we can see that since $\theta$ does not take the value $\pi / 4$ we can bound the first term appropriately. In the second term the maximum value of $\cos \theta$ can be used to bounded it appropriately. This is given below as follows.

$$
\begin{aligned}
\frac{V_{\oplus}^{\prime}}{V_{\oplus}} \leq & \frac{4}{\sqrt{\pi}} \frac{\frac{n}{2}\left(1+\Gamma\left(\frac{n+1}{2}\right)\right) \tan \left(\frac{\pi}{3}+\eta\right)}{\Gamma\left(\frac{n+1}{2}\right)}\left(\sin \left(\frac{\pi}{2}-2 \epsilon\right)\right)^{n-1} \frac{\pi}{2} \\
& +\frac{4}{\sqrt{\pi}} \frac{(n-1) \frac{n}{2}\left(1+\Gamma\left(\frac{n+1}{2}\right)\right)}{\Gamma\left(\frac{n+1}{2}\right)} \frac{\pi}{2}\left[2 \cos \left(\frac{\pi}{3}+\eta\right)\right]^{n-1} \frac{\pi}{2}
\end{aligned}
$$

From the above we can easily see, that both terms tend to 0 as $n \rightarrow \infty$. From this the lemma follows. 


\section{APPENDIX C}

Application of BLiChFEldT's Principle to Show Existence of Good Translations

Lemma 13: Let $\Lambda_{n}$ be a lattice having a fundamental region $P_{n}$ and let $\operatorname{det}\left(\Lambda_{n}\right)$ be it's fundamental volume and define

$$
\begin{array}{r}
P_{n}^{*}=\left\{\left(\mathbf{s}_{\mathbf{1}}, \mathbf{s}_{\mathbf{2}}\right) \in P_{n} \times P_{n}: M_{1}\left(\Lambda_{n}, \mathbf{s}_{\mathbf{1}}\right) \geq \frac{V_{n}(\sqrt{n P})}{8 \operatorname{det}\left(\Lambda_{n}\right)} ;\right. \\
\left.M_{2}\left(\Lambda_{n}, \mathbf{s}_{\mathbf{2}}\right) \geq \frac{V_{n}(\sqrt{n P})}{8 \operatorname{det}\left(\Lambda_{n}\right)} ; \frac{M_{\oplus}^{\prime}\left(\Lambda_{n}, \mathbf{s}_{\mathbf{1}}, \mathbf{s}_{\mathbf{2}}\right)}{M_{\oplus}\left(\Lambda_{n}, \mathbf{s}_{\mathbf{1}}, \mathbf{s}_{\mathbf{2}}\right)} \leq 4 \frac{V_{\oplus}^{\prime}}{V_{\oplus}}\right\}
\end{array}
$$

Then

$$
V_{\oplus} \leq 2 \int_{P_{n}^{*}} M_{\oplus}\left(\Lambda_{n}, \mathbf{s}_{\mathbf{1}}, \mathbf{s}_{\mathbf{2}}\right) d V\left(\mathbf{s}_{\mathbf{1}}, \mathbf{s}_{\mathbf{2}}\right),
$$

where $\delta_{n}>0$ and can be made arbitrarily small for sufficiently large $n$

Proof: Let us define the following sets,

$$
\begin{gathered}
F_{n}=\left\{\mathbf{s}_{\mathbf{1}} \in P_{n}: M_{1}\left(\Lambda_{n}, \mathbf{s}_{\mathbf{1}}\right) \geq \frac{V_{n}(\sqrt{n P})}{8 \operatorname{det}\left(\Lambda_{n}\right)}\right\} \\
G_{n}=\left\{\mathbf{s}_{\mathbf{2}} \in P_{n}: M_{2}\left(\Lambda_{n}, \mathbf{s}_{\mathbf{2}}\right) \geq \frac{V_{n}(\sqrt{n P})}{8 \operatorname{det}\left(\Lambda_{n}\right)}\right\} \\
O_{n}=\left\{\left(\mathbf{s}_{\mathbf{1}}, \mathbf{s}_{\mathbf{2}}\right) \in P_{n} \times P_{n}: \frac{M_{\oplus}^{\prime}\left(\Lambda_{n}, \mathbf{s}_{\mathbf{1}}, \mathbf{s}_{\mathbf{2}}\right)}{M_{\oplus}\left(\Lambda_{n}, \mathbf{s}_{\mathbf{1}}, \mathbf{s}_{\mathbf{2}}\right)} \leq 4 \frac{V_{\oplus}^{\prime}}{V_{\oplus}}\right\}
\end{gathered}
$$

Therefore $P_{n}^{*}=\left(F_{n} \times G_{n}\right) \bigcap O_{n}$. Define the complements here. Hence

$$
P_{n} \times P_{n}=P_{n}^{*} \bigcup\left\{\left(F_{n} \times G_{n}\right) \cap O_{n}^{C}\right\} \bigcup\left\{\left(F_{n} \times G_{n}\right)^{C}\right\}
$$

Hence we have

$$
\begin{gathered}
V_{\oplus}=\int_{P_{n} \times P_{n}} M_{\oplus}\left(\Lambda_{n}, \mathbf{s}_{\mathbf{1}}, \mathbf{s}_{\mathbf{2}}\right) d V\left(\mathbf{s}_{\mathbf{1}}\right) d V\left(\mathbf{s}_{\mathbf{2}}\right) \\
V_{\oplus} \leq \int_{P_{n}^{*}} M_{\oplus}\left(\Lambda_{n}, \mathbf{s}_{\mathbf{1}}, \mathbf{s}_{\mathbf{2}}\right) d V\left(\mathbf{s}_{\mathbf{1}}\right) d V\left(\mathbf{s}_{\mathbf{2}}\right)+\int_{O_{n}^{C}} M_{\oplus}\left(\Lambda_{n}, \mathbf{s}_{\mathbf{1}}, \mathbf{s}_{\mathbf{2}}\right) d V\left(\mathbf{s}_{\mathbf{1}}\right) d V\left(\mathbf{s}_{\mathbf{2}}\right) \\
+\int_{\left(F_{n} \times G_{n}\right)^{C}} M_{\oplus}\left(\Lambda_{n}, \mathbf{s}_{\mathbf{1}}, \mathbf{s}_{\mathbf{2}}\right) d V\left(\mathbf{s}_{\mathbf{1}}\right) d V\left(\mathbf{s}_{\mathbf{2}}\right)
\end{gathered}
$$

We can replace the second integral, using the condition that the translations are not in the set $O_{n}$, to get a bound on $M_{\oplus}$ as shown below,

$$
\begin{aligned}
V_{\oplus} \leq & \int_{P_{n}^{*}} M_{\oplus}\left(\Lambda_{n}, \mathbf{s}_{\mathbf{1}}, \mathbf{s}_{\mathbf{2}}\right) d V\left(\mathbf{s}_{\mathbf{1}}\right) d V\left(\mathbf{s}_{\mathbf{2}}\right)+\frac{V_{\oplus}}{4 V_{\oplus}^{\prime}} \int_{O_{n}^{C}} M_{\oplus}^{\prime}\left(\Lambda_{n}, \mathbf{s}_{\mathbf{1}}, \mathbf{s}_{\mathbf{2}}\right) d V\left(\mathbf{s}_{\mathbf{1}}\right) d V\left(\mathbf{s}_{\mathbf{2}}\right) \\
& +\int_{\left(F_{n} \times G_{n}\right)^{C}}\left(\sum_{h_{1} \in \Lambda_{n}} \sum_{h_{2} \in \Lambda_{n}} f\left(h_{1}+\mathbf{s}_{\mathbf{1}}, h_{2}+\mathbf{s}_{\mathbf{2}}\right)\right) d V\left(\mathbf{s}_{\mathbf{1}}\right) d V\left(\mathbf{s}_{\mathbf{2}}\right)
\end{aligned}
$$

Since, $O_{n}^{C} \subset P_{n} \times P_{n}$, we can bound the second integral again as shown below.

$$
\begin{aligned}
V_{\oplus} \leq & \int_{P_{n}^{*}} M_{\oplus}\left(\Lambda_{n}, \mathbf{s}_{\mathbf{1}}, \mathbf{s}_{\mathbf{2}}\right) d V\left(\mathbf{s}_{\mathbf{1}}\right) d V\left(\mathbf{s}_{\mathbf{2}}\right)+\frac{V_{\oplus}}{4 V_{\oplus}^{\prime}} \int_{P_{n} \times P_{n}} M_{\oplus}^{\prime}\left(\Lambda_{n}, \mathbf{s}_{\mathbf{1}}, \mathbf{s}_{\mathbf{2}}\right) d V\left(\mathbf{s}_{\mathbf{1}}\right) d V\left(\mathbf{s}_{\mathbf{2}}\right) \\
& +\int_{\left(F_{n} \times G_{n}\right)^{C}}\left(\sum_{h_{1} \in \Lambda_{n}} \sum_{h_{2} \in \Lambda_{n}} \chi_{T}\left(h_{1}+\mathbf{s}_{\mathbf{1}}\right) \chi_{T}\left(h_{2}+\mathbf{s}_{\mathbf{2}}\right)\right) d V\left(\mathbf{s}_{\mathbf{1}}\right) d V\left(\mathbf{s}_{\mathbf{2}}\right)
\end{aligned}
$$

By using Blichfeldt's principle and following similar steps as in Corollary 4, we can show 


$$
\begin{aligned}
\int_{P_{n} \times P_{n}} M_{\oplus}^{\prime}\left(\Lambda_{n}, \mathbf{s}_{\mathbf{1}}, \mathbf{s}_{\mathbf{2}}\right) & d V\left(\mathbf{s}_{\mathbf{1}}\right) d V\left(\mathbf{s}_{\mathbf{2}}\right)=V_{\oplus}^{\prime} \\
V_{\oplus} \leq & \int_{P_{n}^{*}} M_{\oplus}^{\Delta}\left(\Lambda_{n}, \mathbf{s}_{\mathbf{1}}, \mathbf{s}_{\mathbf{2}}\right) d V\left(\mathbf{s}_{\mathbf{1}}\right) d V\left(\mathbf{s}_{\mathbf{2}}\right)+\frac{V_{\oplus}}{4} \\
& +\int_{\left(F_{n} \times G_{n}\right)^{C}}\left(\sum_{h_{1} \in \Lambda_{n}} \sum_{h_{2} \in \Lambda_{n}} \chi_{T}\left(h_{1}+\mathbf{s}_{\mathbf{1}}\right) \chi_{T}\left(h_{2}+\mathbf{s}_{\mathbf{2}}\right)\right) d V\left(\mathbf{s}_{\mathbf{1}}\right) d V\left(\mathbf{s}_{\mathbf{2}}\right)
\end{aligned}
$$

Next we change the double summation into a product of two summations, to get

$$
\begin{aligned}
V_{\oplus} \leq & \int_{P_{n}^{*}} M_{\oplus}^{\Delta}\left(\Lambda_{n}, \mathbf{s}_{\mathbf{1}}, \mathbf{s}_{\mathbf{2}}\right) d V\left(\mathbf{s}_{\mathbf{1}}\right) d V\left(\mathbf{s}_{\mathbf{2}}\right)+\frac{V_{\oplus}}{4} \\
& +\int_{\left(F_{n} \times G_{n}\right)^{C}}\left(\sum_{h_{1} \in \Lambda_{n}} \chi_{T}\left(h_{1}+\mathbf{s}_{\mathbf{1}}\right)\right)\left(\sum_{h_{2} \in \Lambda_{n}} \chi_{T}\left(h_{2}+\mathbf{s}_{\mathbf{2}}\right)\right) d V\left(\mathbf{s}_{\mathbf{1}}\right) d V\left(\mathbf{s}_{\mathbf{2}}\right)
\end{aligned}
$$

The summation $\sum_{h_{1} \in \Lambda_{n}} \chi_{T}\left(h_{1}+\mathbf{s}_{1}\right)$ can be seen to count the number of lattice points in the sphere $T_{n}$, for the translation $\mathbf{s}_{1}$. This is by definition equivalent to $M\left(\Lambda_{n}, \mathbf{s}_{1}\right)$. Similarly we can replace the other summation by $M\left(\Lambda_{n}, \mathbf{s}_{\mathbf{2}}\right)$, to get

$$
V_{\oplus} \leq \int_{P_{n}^{*}} M_{\oplus}^{\Delta}\left(\Lambda_{n}, \mathbf{s}_{\mathbf{1}}, \mathbf{s}_{\mathbf{2}}\right) d V\left(\mathbf{s}_{\mathbf{1}}\right) d V\left(\mathbf{s}_{\mathbf{2}}\right)+\frac{V_{\oplus}}{4}+\int_{\left(F_{n} \times G_{n}\right)^{C}} M\left(\Lambda_{n}, \mathbf{s}_{\mathbf{1}}\right) M\left(\Lambda_{n}, \mathbf{s}_{\mathbf{2}}\right) d V\left(\mathbf{s}_{\mathbf{1}}\right) d V\left(\mathbf{s}_{\mathbf{2}}\right)
$$

Since $\left(F_{n} \times G_{n}\right)^{C} \subset\left(F_{n} \times P_{n}\right) \cup\left(P_{n} \times G_{n}\right)$ we can bound the integral to get

$$
\begin{aligned}
V_{\oplus} \leq & \int_{P_{n}^{*}} M_{\oplus}^{\Delta}\left(\Lambda_{n}, \mathbf{s}_{\mathbf{1}}, \mathbf{s}_{\mathbf{2}}\right) d V\left(\mathbf{s}_{\mathbf{1}}\right) d V\left(\mathbf{s}_{\mathbf{2}}\right)+\frac{V_{\oplus}}{4}+\int_{\left(F_{n}^{C} \times P_{n}\right)} M\left(\Lambda_{n}, \mathbf{s}_{\mathbf{1}}\right) M\left(\Lambda_{n}, \mathbf{s}_{\mathbf{2}}\right) d V\left(\mathbf{s}_{\mathbf{1}}\right) d V\left(\mathbf{s}_{\mathbf{2}}\right) \\
& +\int_{\left(P_{n} \times G_{n}^{C}\right)} M\left(\Lambda_{n}, \mathbf{s}_{\mathbf{1}}\right) M\left(\Lambda_{n}, \mathbf{s}_{\mathbf{2}}\right) d V\left(\mathbf{s}_{\mathbf{1}}\right) d V\left(\mathbf{s}_{\mathbf{2}}\right)
\end{aligned}
$$

We next use Blichfeldt's principle to simplify the integrals.

$$
\begin{aligned}
V_{\oplus} \leq & \int_{P_{n}^{*}} M_{\oplus}^{\Delta}\left(\Lambda_{n}, \mathbf{s}_{\mathbf{1}}, \mathbf{s}_{\mathbf{2}}\right) d V\left(\mathbf{s}_{\mathbf{1}}\right) d V\left(\mathbf{s}_{\mathbf{2}}\right)+\frac{V_{\oplus}}{4}+V_{n}(\sqrt{n P}) \int_{F_{n}^{C}} M\left(\Lambda_{n}, \mathbf{s}_{\mathbf{1}}\right) d V\left(\mathbf{s}_{\mathbf{1}}\right) \\
& +V_{n}(\sqrt{n P}) \int_{G_{n}^{C}} M\left(\Lambda_{n}, \mathbf{s}_{\mathbf{2}}\right) d V\left(\mathbf{s}_{\mathbf{2}}\right)
\end{aligned}
$$

We use the condition $\mathbf{s}_{\mathbf{1}} \notin F_{n}$ and $\mathbf{s}_{\mathbf{2}} \notin G_{n}$, to get

$$
\begin{aligned}
V_{\oplus} \leq & \int_{P_{n}^{*}} M_{\oplus}\left(\Lambda_{n}, \mathbf{s}_{\mathbf{1}}, \mathbf{s}_{\mathbf{2}}\right) d V\left(\mathbf{s}_{\mathbf{1}}\right) d V\left(\mathbf{s}_{\mathbf{2}}\right)+\frac{V_{\oplus}}{4}+V_{n}(\sqrt{n P}) \frac{V_{n}(\sqrt{n P})}{8 \operatorname{det}\left(\Lambda_{n}\right)} \int_{F_{n}^{C}} d V\left(\mathbf{s}_{\mathbf{1}}\right) \\
& +V_{n}(\sqrt{n P}) \frac{V_{n}(\sqrt{n P})}{8 \operatorname{det}\left(\Lambda_{n}\right)} \int_{G_{n}^{C}} d V\left(\mathbf{s}_{\mathbf{2}}\right)
\end{aligned}
$$

Since $F_{n}^{C}, G_{n}^{C} \subset P_{n}$ and $\int_{P_{n}} d V\left(\mathbf{s}_{\mathbf{1}}\right), \int_{P_{n}} d V\left(\mathbf{s}_{\mathbf{2}}\right)=\operatorname{det}\left(\Lambda_{n}\right)$, we obtain,

$$
V_{\oplus} \leq \int_{P_{n}^{*}} M_{\oplus}\left(\Lambda_{n}, \mathbf{s}_{\mathbf{1}}, \mathbf{s}_{\mathbf{2}}\right) d V\left(\mathbf{s}_{\mathbf{1}}\right) d V\left(\mathbf{s}_{\mathbf{2}}\right)+\frac{V_{\oplus}}{4}+V_{n}(\sqrt{n P}) \frac{V_{n}(\sqrt{n P})}{8}+V_{n}(\sqrt{n P}) \frac{V_{n}(\sqrt{n P})}{8}
$$

Finally using $V_{\oplus}=\left(V_{n}(\sqrt{n P})\right)^{2}$, we obtain

$$
V_{\oplus} \leq 2 \int_{P_{n}^{*}} M_{\oplus}\left(\Lambda_{n}, \mathbf{s}_{\mathbf{1}}, \mathbf{s}_{\mathbf{2}}\right) d V\left(\mathbf{s}_{\mathbf{1}}\right) d V\left(\mathbf{s}_{\mathbf{2}}\right)
$$

From the above lemma it can be seen that the measure of $P_{n}^{*}$ must be non-zero and hence, there must be at least some translations $\left(\mathbf{s}_{\mathbf{1}}, \mathbf{s}_{\mathbf{2}}\right)$ of the lattice, where the requirements hold. 


\section{APPENDIX D}

MinkowsKi-Hlawka TheOREM TO SHOw GoOd LATTICES EXIST

Lemma 14: There exists translational vectors $\mathbf{s}_{1}^{*}, \mathbf{s}_{2}^{*}$ such that the following holds

$$
\begin{array}{r}
\frac{1}{M_{\oplus}\left(\Lambda_{n}, \mathbf{s}_{\mathbf{1}}^{*}, \mathbf{s}_{\mathbf{2}}^{*}\right)} \sum_{\left(\mathbf{x}_{\mathbf{1}}, \mathbf{x}_{\mathbf{2}}\right) \in \mathcal{C}_{\oplus}} \sum_{g \in \Lambda_{n} \backslash\{0\}} p_{\theta}\left(\mathbf{x}_{\mathbf{1}}+\mathbf{x}_{\mathbf{2}}, g+\mathbf{x}_{\mathbf{1}}+\mathbf{x}_{\mathbf{2}}\right) \chi_{T_{\sqrt{2 P}}^{\Delta}}\left(g+\mathbf{x}_{\mathbf{1}}+\mathbf{x}_{\mathbf{2}}\right) \\
\leq 2 \frac{(n-1) \pi^{\frac{n-1}{2}}(n(2 P+\delta))^{n / 2}}{d_{n} n \Gamma\left(\frac{n+1}{2}\right)} \int_{0}^{\theta} \sin ^{n-2}(x) d x
\end{array}
$$

and

Proof:

$$
M_{1}\left(\Lambda_{n}, \mathbf{s}_{\mathbf{1}}^{*}\right) \geq \frac{V_{n}(\sqrt{n P})}{8 \operatorname{det}\left(\Lambda_{n}\right)} ; M_{2}\left(\Lambda_{n}, \mathbf{s}_{\mathbf{2}}^{*}\right) \geq \frac{V_{n}(\sqrt{n P})}{8 \operatorname{det}\left(\Lambda_{n}\right)} ; \frac{M_{\oplus}^{\prime}\left(\Lambda_{n}, \mathbf{s}_{\mathbf{1}}^{*}, \mathbf{s}_{\mathbf{2}}^{*}\right)}{M_{\oplus}\left(\Lambda_{n}, \mathbf{s}_{\mathbf{1}}^{*}, \mathbf{s}_{\mathbf{2}}^{*}\right)} \leq 4 \frac{V_{\oplus}^{\prime}}{V_{\oplus}}
$$

First let us define the function $f(z)$ as follows

$$
f(\mathbf{z})=\int_{\mathbb{R}^{n}} \int_{\mathbb{R}^{n}} p_{\theta}(\mathbf{u}+\mathbf{v}, \mathbf{z}+\mathbf{u}+\mathbf{v}) \chi_{T_{\sqrt{2 P}}^{\Delta}}(g+\mathbf{u}+\mathbf{v}) \chi_{T}(\mathbf{u}) \chi_{T}(\mathbf{v}) \chi_{T_{\sqrt{2 P}}^{\Delta}}(\mathbf{u}+\mathbf{v}) d V(\mathbf{u}, \mathbf{v})
$$

Clearly $f(z)$ is a nonnegative integrable function and has bounded support. Hence we can apply the MinkowskiHlawka theorem and find a lattice $\Lambda_{n}^{*}$, such that

$$
\sum_{g \in \Lambda_{n} \backslash\{0\}} f(g) \leq \frac{1}{d_{n}} \int_{\mathbb{R}^{n}} f(\mathbf{z}) d V(\mathbf{z})
$$

Consider the integral

$$
\begin{aligned}
I= & \int_{P_{n}^{*}} M_{\oplus}\left(\Lambda_{n}, \mathbf{s}_{\mathbf{1}}, \mathbf{s}_{\mathbf{2}}\right)\left[\frac{1}{M_{\oplus}\left(\Lambda_{n}, \mathbf{s}_{\mathbf{1}}, \mathbf{s}_{\mathbf{2}}\right)} \sum_{\left(\mathbf{x}_{\mathbf{1}}, \mathbf{x}_{\mathbf{2}}\right) \in \mathcal{C}_{\oplus}} \sum_{g \in \Lambda_{n} \backslash\{0\}} p_{\theta}\left(\mathbf{x}_{\mathbf{1}}+\mathbf{x}_{\mathbf{2}}, g+\mathbf{x}_{\mathbf{1}}+\mathbf{x}_{\mathbf{2}}\right)\right. \\
& \left.\chi_{T_{\sqrt{2 P}}^{\Delta}}\left(g+\mathbf{x}_{\mathbf{1}}+\mathbf{x}_{\mathbf{2}}\right)\right] d V\left(\mathbf{s}_{\mathbf{1}}, \mathbf{s}_{\mathbf{2}}\right) \\
I= & \int_{P_{n}^{*}} \sum_{\left(\mathbf{x}_{\mathbf{1}}, \mathbf{x}_{\mathbf{2}}\right) \in \mathcal{C}_{\oplus}^{\Delta}} \sum_{g \in \Lambda_{n} \backslash\{0\}} p_{\theta}\left(\mathbf{x}_{\mathbf{1}}+\mathbf{x}_{\mathbf{2}}, g+\mathbf{x}_{\mathbf{1}}+\mathbf{x}_{\mathbf{2}}\right) \chi_{T_{\sqrt{2 P}}^{\Delta}}\left(g+\mathbf{x}_{\mathbf{1}}+\mathbf{x}_{\mathbf{2}}\right) d V\left(\mathbf{s}_{\mathbf{1}}, \mathbf{s}_{\mathbf{2}}\right) \\
\leq & \int_{P_{n} \times P_{n}} \sum_{\left(\mathbf{x}_{\mathbf{1}}, \mathbf{x}_{\mathbf{2}}\right) \in \mathcal{C}_{\oplus}^{\Delta}} \sum_{g \in \Lambda_{n} \backslash\{0\}} p_{\theta}\left(\mathbf{x}_{\mathbf{1}}+\mathbf{x}_{\mathbf{2}}, g+\mathbf{x}_{\mathbf{1}}+\mathbf{x}_{\mathbf{2}}\right) \chi_{T_{\sqrt{2 P}}^{\Delta}}\left(g+\mathbf{x}_{\mathbf{1}}+\mathbf{x}_{\mathbf{2}}\right) d V\left(\mathbf{s}_{\mathbf{1}}, \mathbf{s}_{\mathbf{2}}\right)
\end{aligned}
$$

The above follows as $P_{n}^{*} \subseteq P_{n} \times P_{n}$.

$$
\begin{aligned}
& I \leq \int_{P_{n} \times P_{n}} \sum_{\left(\mathbf{h}_{\mathbf{1}}, \mathbf{h}_{\mathbf{2}}\right) \in \Lambda_{n} \times \Lambda_{n}} \sum_{g \in \Lambda_{n} \backslash\{0\}} p_{\theta}\left(h_{1}+\mathbf{s}_{\mathbf{1}}+h_{2}+\mathbf{s}_{\mathbf{2}}, g+h_{1}+\mathbf{s}_{\mathbf{1}}+h_{2}+\mathbf{s}_{\mathbf{2}}\right) \\
& \chi_{T_{\sqrt{2 P}}^{\Delta}}\left(g+h_{1}+\mathbf{s}_{\mathbf{1}}+h_{2}+\mathbf{s}_{\mathbf{2}}\right) \chi_{T}\left(h_{1}+\mathbf{s}_{\mathbf{1}}\right) \chi_{T}\left(h_{2}+\mathbf{s}_{\mathbf{2}}\right) \chi_{T_{\sqrt{2 P}}^{\Delta}}\left(h_{1}+\mathbf{s}_{\mathbf{1}}+h_{2}+\mathbf{s}_{\mathbf{2}}\right) d V\left(\mathbf{s}_{\mathbf{1}}, \mathbf{s}_{\mathbf{2}}\right)
\end{aligned}
$$

In the above equation we substituted $\mathbf{x}_{\mathbf{1}}=h_{1}+\mathbf{s}_{\mathbf{1}}$ and $\mathbf{x}_{\mathbf{2}}=h_{2}+\mathbf{s}_{\mathbf{2}}$. Next applying the Blichfeldt's principle twice we obtain,

$$
I \leq \int_{\mathbb{R}^{n}} \int_{\mathbb{R}^{n}} \sum_{g \in \Lambda_{n} \backslash\{0\}} p_{\theta}(\mathbf{u}+\mathbf{v}, g+\mathbf{u}+\mathbf{v}) \chi_{T_{\sqrt{2 P}}^{\Delta}}(g+\mathbf{u}+\mathbf{v}) \chi_{T}(\mathbf{u}) \chi_{T}(\mathbf{v}) \chi_{T_{\sqrt{2 P}}^{\Delta}}(\mathbf{u}+\mathbf{v}) d V(\mathbf{u}, \mathbf{v})
$$

Here $\mathbf{u}=h_{1}+\mathbf{s}_{\mathbf{1}}$ and $\mathbf{v}=h_{2}+\mathbf{s}_{\mathbf{2}}$. We can next take the summation outside the double integral, as we are dealing with a finite number of non-zero sums. This gives,

$$
I \leq \sum_{g \in \Lambda_{n} \backslash\{0\}} \int_{\mathbb{R}^{n}} \int_{\mathbb{R}^{n}} p_{\theta}(\mathbf{u}+\mathbf{v}, g+\mathbf{u}+\mathbf{v}) \chi_{T_{\sqrt{2 P}}^{\Delta}}(g+\mathbf{u}+\mathbf{v}) \chi_{T}(\mathbf{u}) \chi_{T}(\mathbf{v}) \chi_{T_{\sqrt{2 P}}^{\Delta}}(\mathbf{u}+\mathbf{v}) d V(\mathbf{u}, \mathbf{v})
$$


Next we use the Minkowski-Hlawka theorem to establish that there exists at least one lattice $\Lambda_{n}^{*}$ such that the summation can be bounded by an integral, as shown below. In short the Minkowski-Hlawka theorem gives an existence result that such a lattice can be found, but does not give a way to find such a lattice.

$$
I \leq \frac{1}{d_{n}} \iint_{\mathbb{R}^{n}} \int_{\mathbb{R}^{n}} p_{\theta}(\mathbf{u}+\mathbf{v}, \mathbf{w}) \chi_{T_{\sqrt{2 P}}^{\Delta}}(\mathbf{w}) \chi_{T}(\mathbf{u}) \chi_{T}(\mathbf{v}) \chi_{T_{\sqrt{2 P}}^{\Delta}}(\mathbf{u}+\mathbf{v}) d V(\mathbf{u}, \mathbf{v}) d V(\mathbf{w})
$$

In the above we have replaced $\mathbf{w}+\mathbf{u}+\mathbf{v}$ with $\mathbf{w}$. Again we change the order of integration to get,

$$
I \leq \frac{1}{d_{n}} \int_{\mathbb{R}^{n}} \int_{\mathbb{R}^{n}}\left[\int p_{\theta}(\mathbf{u}+\mathbf{v}, \mathbf{w}) \chi_{T_{\sqrt{2 P}}^{\Delta}}(\mathbf{w}) d V(\mathbf{w})\right] \chi_{T}(\mathbf{u}) \chi_{T}(\mathbf{v}) \chi_{T_{\sqrt{2 P}}^{\Delta}}(\mathbf{u}+\mathbf{v}) d V(\mathbf{u}, \mathbf{v})
$$

The inner integral can be seen to be independent of $\mathbf{u}+\mathbf{v}$, and the outer integral can be seen to be lesser than $V_{\oplus}$. Hence we can express the resultant integral as,

$$
I \leq \frac{V_{\oplus}}{d_{n}} \int p_{\theta}\left(\mathbf{s}_{\mathbf{0}}, \mathbf{w}\right) \chi_{T_{\sqrt{2 P}}^{\Delta}}(\mathbf{w}) d V(\mathbf{w}) .
$$

Hence we next evaluate the integral using similar steps as in [11], first by changing to spherical coordinates.

$$
I \leq \frac{V_{\oplus}}{d_{n}} \int_{\sqrt{n(2 P-\delta)}}^{\sqrt{n(2 P+\delta)}}\left[\int_{\mathbf{w}:\|\mathbf{w}\|=r} p_{\theta}\left(\mathbf{s}_{\mathbf{0}}, \mathbf{w}\right) \chi_{T_{\sqrt{2 P}}^{\Delta}}(\mathbf{w}) d A(\mathbf{w})\right] d r,
$$

where $d A(\mathbf{w})$ is the $(n-1)$-dimensional volume element of a thin spherical element at a distance $r$ from the origin. Next we use the definition of $p_{\theta}$ to get,

$$
I \leq \frac{V_{\oplus}}{d_{n}} \int_{\sqrt{n(2 P-\delta)}}^{\sqrt{n(2 P+\delta)}}\left[\int_{\mathbf{w}:\|\mathbf{w}\|=r} \operatorname{Pr}\left(\pi\left(\mathbf{s}_{\mathbf{0}}\right)+Z^{n} \in B_{\theta}(\mathbf{w})\right) \chi_{T_{\sqrt{2 P}}^{\Delta}}(\mathbf{w}) d A(\mathbf{w})\right] d r .
$$

We evaluate the probability by conditioning on $Z^{n}$.

$$
\begin{array}{r}
I \leq \frac{V_{\oplus}}{d_{n}} \int_{\sqrt{n(2 P-\delta)}}^{\sqrt{n(2 P+\delta)}}\left[\int_{\mathbf{w}:\|\mathbf{w}\|=r}\left[\int \operatorname{Pr}\left(\pi\left(\mathbf{s}_{\mathbf{0}}\right)+Z^{n} \in B_{\theta}(\mathbf{w}) \mid Z^{n}=\mathbf{z}\right) \operatorname{Pr}\left(Z^{n}=\mathbf{z}\right) d V(\mathbf{z})\right]\right. \\
\left.\chi_{T_{\sqrt{2 P}}^{\Delta}}(\mathbf{w}) d A(\mathbf{w})\right] d r .
\end{array}
$$

Since the function is non-negative, the order of integration can be interchanged to obtain

$$
\begin{array}{r}
I \leq \frac{V_{\oplus}}{d_{n}} \int\left[\int_{\sqrt{n(2 P-\delta)}}^{\sqrt{n(2 P+\delta)}} \int_{\mathbf{w}:\|\mathbf{w}\|=r}\left[\operatorname{Pr}\left(\pi\left(\mathbf{s}_{\mathbf{0}}\right)+Z^{n} \in B_{\theta}(\mathbf{w}) \mid Z^{n}=\mathbf{z}\right)\right]\right. \\
\left.\chi_{T_{\sqrt{2 P}}^{\Delta}}(\mathbf{w}) d A(\mathbf{w}) d r\right] \operatorname{Pr}\left(Z^{n}=\mathbf{z}\right) d V(\mathbf{z}) .
\end{array}
$$

Due to the conditioning on $Z^{n}$, the conditional probability becomes deterministic and is equivalent to the cross sectional area of a hyper cone of half-angle $\theta$, intersecting with a hyper-sphere of radius $r$. Note that in the result in [11], the area of cross section must be a function of $r$ and not of $\sqrt{n S^{\prime}}$. This gives,

$$
I \leq \frac{V_{\oplus}}{d_{n}}\left[\int \operatorname{Pr}\left(Z^{n}=\mathbf{z}\right) d V(\mathbf{z})\right] \int_{\sqrt{n(2 P-\delta)}}^{\sqrt{n(2 P+\delta)}}\left[\frac{(n-1) \pi^{\frac{n-1}{2}} r^{n-1}}{\Gamma\left(\frac{n+1}{2}\right)} \int_{0}^{\theta} \sin ^{n-2}(x) d x\right] d r .
$$

This in turn yields

$$
\begin{gathered}
I \leq \frac{V_{\oplus}}{d_{n}}\left[\frac{(n-1) \pi^{\frac{n-1}{2}}\left((n(2 P+\delta))^{n / 2}-(n(2 P-\delta))^{n / 2}\right)}{n \Gamma\left(\frac{n+1}{2}\right)} \int_{0}^{\theta} \sin ^{n-2}(x) d x\right] . \\
I \leq \frac{V_{\oplus}}{d_{n}}\left[\frac{(n-1) \pi^{\frac{n-1}{2}}(n(2 P+\delta))^{n / 2}}{n \Gamma\left(\frac{n+1}{2}\right)} \int_{0}^{\theta} \sin ^{n-2}(x) d x\right] .
\end{gathered}
$$


Next using Lemma 13 we can bound the resultant integral to get,

$$
I \leq 2 \int_{P_{n}^{*}} M_{\oplus}\left(\Lambda_{n}, \mathbf{s}_{\mathbf{1}}, \mathbf{s}_{\mathbf{2}}\right)\left[\frac{(n-1) \pi^{\frac{n-1}{2}}(n(2 P+\delta))^{n / 2}}{d_{n} n \Gamma\left(\frac{n+1}{2}\right)} \int_{0}^{\theta} \sin ^{n-2}(x) d x\right] d V\left(\mathbf{s}_{\mathbf{1}}, \mathbf{s}_{\mathbf{2}}\right) .
$$

Now comparing the integrals and also knowing that the measure of $P_{n}^{*}$ is non-zero we can see that there must be at least one such translational vector pair $\left(\mathbf{s}_{\mathbf{1}}, \mathbf{s}_{\mathbf{2}}\right)$ such that the required assertion of the lemma holds.

\section{REFERENCES}

[1] P. Larsson, N. Johansson and K. -E. Sunell, "Coded bi-directional relaying," The 5th Scandinavian Workshop on Wireless ad-hoc networks, Stockholm, May. 2005.

[2] S. Katti, H. Rahul, W. Hu, D. Katabi, M. Medard and J. Crowcroft, "XORs in the Air: Practical Wireless Network Coding", ACM SIGCOMM 2006.

[3] S. Katti, S. Gollakota and D. Katabi, "Embracing Wireless Interference: Analog Network Coding”, ACM SIGCOMM 2007.

[4] Y. Wu, P. A. Chou and S. Y. Kung, "Information exchange in wireless networks with network coding and physical-layer broadcast," in Proc. of the 2005 Conference on Information Sciences and Systems, The Johns Hopkins University, March 16-18, 2005.

[5] T. J. Oechtering,I. Bjelakovic, C. Schnurr, H. Boche, "Broadcast Capacity Region of Two-Phase Bidirectional Relaying," submitted to IEEE Trans. Inform. Theory,2007.

[6] P. Popovski and H. Yomo, "Physical Network Coding in Two-Way Wireless Relay Channels", Proc. of IEEE -ICC, Glasgow, Scotland, 2007.

[7] F. Xue and S. Sandhu, "PHY-layer network coding for broadcast channel with side information",ITW 2007, Lake Tahoe, CA, September 2007.

[8] J. Körner and K. Marton, "How to encode the modulo-two sum of binary sources," IEEE Tran. Info. Theory, vol. 25, pp. 219 221, March 1979

[9] B. Nazer and M. Gastpar, "Computation over Multiple-Access Channels", to appear at IEEE Tran. Info. Theory, October 2007.

[10] B. Nazer and M. Gastpar, "The Case for Structured Random Codes in Network Communication Theorems", ITW 2007, Lake Tahoe, CA, September 2007.

[11] R. Urbanke and B. Rimoldi, "Lattice codes can achieve capacity on the AWGN channel,", IEEE Tran. Info. Theory, vol. 44, pp. 273278, January 1998.

[12] U. Erez and R. Zamir, "Achieving $\frac{1}{2} \log (1+\mathrm{SNR})$ on the AWGN channel with lattice encoding and decoding," IEEE Tran. Info. Theory, vol. 50, pp. 22932314, October 2004.

[13] R. Zamir, S. Shamai, and U. Erez, "Nested linear/lattice codes for structured multiterminal binning,"IEEE Tran. Info. Theory, vol. 48, pp. 12501276, June 2002.

[14] U. Erez, S. Litsyn, and R. Zamir, "Lattices which are good for (almost) everything,"IEEE Tran. Info. Theory, vol. 51, pp. 34013416, October 2005.

[15] R. De Buda, "Some optimal codes have structure," IEEE Journal on Selected Areas in Communications, vol. 6, pp. 893-899, August 89.

[16] E. Hlawka, J. Schoißengeier, and R. Taschner, Geometric and Analytic Number Theory, New York: Springer Verlag, 1991.

[17] T. Linder, C. Schlegel, and K. Zeger, "Corrected proof of de Budas theorem," IEEE Tran. Info. Theory, vol. 39, pp. 1735-1737, September 93.

[18] C. E. Shannon, "Probability of error for optimal codes in a Gaussian channel," Bell Syst. Tech. J., vol. 38, pp. 611-656, May 1959.

[19] K. R. Narayanan, M. P. Wilson and A. Sprintson, "Joint Physical Layer Coding and Network Coding for Bi-Directional Relaying", 45th Annual Allerton Conference on Communication, Control and Computing, September 2007.

[20] B. Nazer and M. Gastpar, "Lattice Coding Increases Multicast Rates for Gaussian Multiple-Access Networks", 45th Annual Allerton Conference on Communication, Control and Computing, September 2007. 